\title{
Quantum Phase Transition in an Interacting Fermionic Chain.
}

\author{
F. Bonetto ${ }^{1}$ and V. Mastropietro ${ }^{2}$ \\ ${ }^{1}$ School of Mathematics, Georgia Institute of Technology, Atlanta, GA \\ 30332 USA \\ bonetto@math.gatech.edu \\ ${ }^{2}$ Dipartimento di Matematica "Federigo Enriques", Università degli Studi \\ di Milano, \\ Via Cesare Saldini 50, Milano, Italy \\ vieri.mastropietro@unimi.it
}

September 3, 2018

\begin{abstract}
We rigorously analyze the quantum phase transition between a metallic and an insulating phase in (non solvable) interacting spin chains or one dimensional fermionic systems. In particular, we prove the persistence of Luttinger liquid behavior in the presence of an interaction even arbitrarily close to the critical point, where the Fermi velocity vanishes and the two Fermi points coalesce. The analysis is based on two different multiscale analysis; the analysis of the first regime provides gain factors which compensate exactly the small divisors due to the vanishing Fermi velocity.
\end{abstract}

\section{Introduction}

\subsection{Spin or fermionic chains}

Recently a great deal of attention has been focused on the quantum phase transition between a metallic and an insulating phase in (non solvable) interacting spin chains or one dimensional fermionic systems. Beside its intrinsic interest, such problem has a paradigmatic character, see e.g. [1, 2]. Interacting fermionic systems are often investigated using bosonization [3], but such method cannot be used 
in this case; it requires linear dispersion relation, while in our case close to the critical point the dispersion relation becomes quadratic. Interacting fermionic systems with non linear dispersion relation have been studied using convergent expansions, based on rigorous Renormalization Group methods. However the estimate for the radius of convergence of the expansions involved vanishes at the critical point, so that they provide no information close to the quantum phase transition. This paper contains the first rigorous study of the behavior close to the metal insulator transition, using an expansion convergent uniformly in a region of parameters including the critical point.

We will focus for definiteness on the model whose Hamiltonian is given by

$$
H=-\sum_{x} \frac{1}{2}\left[S_{x}^{1} S_{x+1}^{1}+S_{x}^{2} S_{x+1}^{2}\right]-\lambda \sum_{x, y} v(x-y) S_{x}^{3} S_{y}^{3}-\bar{h} \sum_{x} S_{x}^{3}+U_{L}
$$

where $\left(S_{x}^{1}, S_{x}^{2}, S_{x}^{3}\right)=\frac{1}{2}\left(\sigma_{x}^{1}, \sigma_{x}^{2}, \sigma_{x}^{3}\right)$, for $x=1,2, \ldots, L, \sigma_{x}^{i}, i=1,2,3$ are Pauli matrices, $\bar{h}$ is the magnetic field and $v(x)$ is a short range even potential, that is

$$
v(-x)=v(x), \quad|v(x)| \leq e^{-\kappa x} .
$$

Finally $U_{L}$ is an operator depending only on $S_{1}^{i}$ and $S_{L}^{i}$ to be used later to fix the boundary conditions. When $v(x-y)=\delta_{x, y+1}$, this model is known as $X X Z$ Heisenberg spin chain. Setting $\mathbf{x}=\left(x_{0}, x\right)$, we define $S_{\mathbf{x}}^{i}=e^{H x_{0}} S_{x}^{i} e^{-H x_{0}}$. Moreover, given an observable $O$, we define

$$
\langle O\rangle_{\beta, L}=\frac{\operatorname{Tr} e^{-\beta H} O}{\operatorname{Tr} e^{-\beta H}} \quad \text { and } \quad\langle O\rangle=\lim _{\beta \rightarrow \infty} \lim _{L \rightarrow \infty}\langle O\rangle_{\beta, L} .
$$

It is well known that spin chains can be rewritten in terms of fermionic operators $a_{x}^{ \pm}$, with $\left\{a_{x}^{+}, a_{y}^{-}\right\}=\delta_{x, y},\left\{a_{x}^{+}, a_{y}^{+}\right\}=\left\{a_{x}^{-}, a_{y}^{-}\right\}=0$, by the Jordan-Wigner transformation:

$$
\sigma_{x}^{-}=e^{-i \pi \sum_{y=1}^{x-1} a_{y}^{+} a_{y}^{-}} a_{x}^{-}, \quad \sigma_{x}^{+}=a_{x}^{+} e^{i \pi \sum_{y=1}^{x-1} a_{y}^{+} a_{y}^{-}}, \quad \sigma_{x}^{3}=2 a_{x}^{+} a_{x}^{-}-1
$$

where $\sigma_{x}^{ \pm}=\left(\sigma_{x}^{1} \pm i \sigma_{x}^{2}\right) / 2$. In terms of the fermionic operators the Hamiltonian becomes

$$
H=-\sum_{x}\left[\frac{1}{2}\left(a_{x+1}^{+} a_{x}^{-}+a_{x}^{+} a_{x+1}^{-}\right)+h a_{x}^{+} a_{x}^{-}\right]-\lambda \sum_{x, y} v(x-y) a_{x}^{+} a_{x}^{-} a_{y}^{+} a_{y}^{-}
$$

where $h=\bar{h}-\lambda \hat{v}(0)$ and $U_{L}$ can be chosen so to obtain periodic boundary conditions for the fermions, i.e. $a_{L}^{ \pm}=a_{1}^{ \pm}$. Therefore the spin chain (1) can be equivalently represented as a model for interacting spinless fermions in one dimension with chemical potential $\mu=-h$. 
The 2-point Schwinger function is defined as

$$
S_{L, \beta}(\mathbf{x}-\mathbf{y})=\left\langle\mathbf{T} a_{\mathbf{x}}^{-} a_{\mathbf{y}}^{+}\right\rangle_{L, \beta}
$$

where $\mathbf{T}$ is the time ordering operator, that is $\mathbf{T}\left(a_{\mathbf{x}}^{-} a_{\mathbf{y}}^{+}\right)=a_{\mathbf{x}}^{-} a_{\mathbf{y}}^{+}$if $x_{0}>y_{0}$ and $\mathbf{T}\left(a_{\mathbf{x}}^{-} a_{\mathbf{y}}^{+}\right)=-a_{\mathbf{y}}^{+} a_{\mathbf{x}}^{-}$if $x_{0} \leq y_{0}$. We will mostly study the infinite volume zero temperature 2-points Schwinger function given by

$$
\lim _{\beta \rightarrow \infty} \lim _{L \rightarrow \infty} S_{L, \beta}(\mathbf{x}-\mathbf{y})=S(\mathbf{x}-\mathbf{y}) .
$$

\subsection{Quantum Phase transition in the non interacting case}

The fermionic representation makes the analysis of the $\lambda=0$ case (the so called $X X$ chain) quite immediate; writing

$$
a_{x}^{ \pm}=\frac{1}{L} \sum_{k} e^{ \pm i k x} \hat{a}_{k}^{ \pm}
$$

the Hamiltonian becomes

$$
H_{0}=\frac{1}{L} \sum_{k} \varepsilon(k) \hat{a}_{k}^{+} \hat{a}_{k}^{-} \quad \varepsilon(k)=-\cos k-h
$$

where $k=\frac{2 \pi n}{L},-\pi \leq k<\pi$.

The ground state of (6) depends critically on $h$. Indeed, for $h<-1$ the ground state is the fermionic vacuum (empty band insulating state), for $h>1$ it is the state with all fermionic levels occupied (filled band insulating state) and $-1<h<1$ the ground state corresponds to the state in which all the fermionic levels with momenta $|k| \leq p_{F}=\arccos (-h)$, are occupied (metallic state). $p_{F}$ is called Fermi momentum and $\pm p_{F}$ are the Fermi points (the analogous of the Fermi surface in one dimension). In other words the values $h= \pm 1$ separate two different behaviors at zero temperature; one says that in correspondence of $h= \pm 1$ there is a quantum phase transition [1] between a metallic and an insulating phase.

The metallic or insulating phases are signaled by different properties of the two point Schwinger function, which is given by

$$
\begin{aligned}
& S_{0, L, \beta}(\mathbf{x}-\mathbf{y})=\frac{1}{L} \sum_{k} e^{i k x}\left\{\frac{e^{-\left(x_{0}-y_{0}\right) \varepsilon(k)}}{1+e^{-\beta \varepsilon(k)}} \vartheta\left(x_{0}-y_{0}>0\right)-\right. \\
&\left.\frac{e^{-\left(\beta+x_{0}-y_{0}\right) \varepsilon(k)}}{1+e^{-\beta \varepsilon(k)}} \vartheta\left(x_{0}-y_{0} \leq 0\right)\right\}
\end{aligned}
$$


where $\vartheta\left(x_{0}\right)=1$ if $x_{0}>0$ and $\vartheta\left(x_{0}\right)=0$ otherwise. The Schwinger function (7) is defined for $-\beta \leq x_{0} \leq \beta$ but it can be extended periodically over the whole real axis; such extension is smooth in $x_{0}-y_{0}$ for $x_{0}-y_{0} \neq n \beta, n \in \mathbb{Z}$ and it is discontinuous at $\mathbf{x}-\mathbf{y}=(n \beta, 0)$. Since $S_{0, L, \beta}(\mathbf{x})$ is antiperiodic in $x_{0}$ its Fourier series is of the form

$$
S_{0, L, \beta}(\mathbf{x})=\frac{1}{L} \sum_{k} e^{i k x} \hat{S}_{0, L, \beta}\left(k, x_{0}\right)=\frac{1}{\beta L} \sum_{\mathbf{k} \in \mathscr{D}} e^{-i \mathbf{k x}} \hat{S}_{0, \beta, L}(\mathbf{k})
$$

with $\mathbf{k}=\left(k_{0}, k\right), \mathscr{D}=\left\{\mathbf{k} \mid k=\frac{2 \pi m}{L},-\pi \leq k<\pi, k_{0}=\frac{2 \pi}{\beta}\left(n+\frac{1}{2}\right)\right\}$ and

$$
\hat{S}_{0, L, \beta}(\mathbf{k})=\frac{1}{-i k_{0}+\cos k+h}
$$

In the metallic phase the Schwinger function $\hat{S}_{0}(\mathbf{k})$ is singular in correspondence of the Fermi points $\left(0, \pm p_{F}\right)$. For $|k|$ close to $p_{F}$ we have $\hat{S}_{0}(\mathbf{k}) \sim \frac{1}{-i k_{0}+v_{F}\left(k \mid-p_{F}\right)}$. Notice that the 2-point Schwinger function is asymptotically identical, if the momenta are measured from the Fermi points, to the Schwinger function of massless Dirac fermions in $d=1+1$ with Fermi velocity $v_{F}$. For values of $h$ close to $h=-1$ (i.e. for small positive $r$ if we set $h=-1+r$ ) both the distance of the Fermi points and $v_{F}$ are $O(\sqrt{r})$, that is the Fermi velocity vanishes with continuity and the two Fermi points coalesce. At criticality when $r=0$ the 2-point function $\hat{S}_{0}(\mathbf{k})$ is singular only at $(0,0)$ and $\hat{S}_{0}(\mathbf{k}) \sim \frac{1}{-i k_{0}+\frac{1}{2} k^{2}}$; the elementary excitations do not have a relativistic linear dispersion relation, as in the metallic phase, but a parabolic one. Finally in the insulating phase for $r<0$ the two point function has no singularities.

It is natural to ask what happens to the quantum phase transition in presence of the interaction.

\subsection{Quantum Phase transition in the interacting case}

The Schwinger functions of the interacting model in the metallic phase have been constructed using Renormalization Group (RG) methods in [4, 5, 6]. Luttinger liquid behavior (in the sense of [7]) has been established, showing that the power law decay of correlations is modified by the interaction via the appearance of critical exponents, that depend in a non trivial way on the interaction. It should be stressed that such analysis provides a full understanding inside the metallic phase, but gives no information on the phase transition; the reason is that the physical observables are expressed in terms of renormalized expansions which are convergent under the condition

$$
|\lambda| \leq \varepsilon\left|v_{F}\right|
$$


and small $\varepsilon$; therefore, the closer one is to the bottom (or the top) of the band, the smaller the interaction has to be chosen. This is not surprising, as such RG methods essentially show that the interacting fermionic chain is asymptotic to a system of interacting massless Dirac fermions in $d=1+1$ dimensions with coupling $\frac{\lambda}{v_{F}}$. One may even suspect that an extremely weak interaction could produce some quantum instability close to the boundary of the metallic phase, where the parameters correspond to a strong coupling regime in the effective description.

This is however excluded by our results; we prove the persistence of the metallic phase, with Luttinger liquid behavior, in presence of interaction even arbitrarily close to the critical point, where the Fermi velocity vanishes. This result is achieved writing the correlations in terms of a renormalized expansion with a radius of convergence which is independent from the Fermi velocity. In order to obtain this result we needs to exploit the non linear corrections to the dispersion relation due to the lattice. The proof is indeed based on two different multiscale analysis in two regions of the energy momentum space; in the smaller energy region the effective relativistic description is valid while in the larger energy region the quadratic corrections due to the lattice are dominating. The scaling dimensions in the two regimes are different; after the integration of the first regime one gets gain factors which compensate exactly the velocities at the denominator produced in the second regime, so that uniformity is achieved.

Our main results are summarized by the following theorem. We state it in terms of the Fourier transform of the 2-points Schwinger function defined by

$$
S_{L, \beta}(\mathbf{x})=\frac{1}{\beta L} \sum_{\mathbf{k} \in \mathscr{D}} e^{i \mathbf{k} \mathbf{x}} \hat{S}_{L, \beta}(\mathbf{k}) \quad \hat{S}(\mathbf{k})=\lim _{\beta \rightarrow \infty} \lim _{L \rightarrow \infty} \hat{S}_{L, \beta}(\mathbf{k}) .
$$

Theorem 1.1. Given $h=-1+r$ with $|r|<1$, there exists $\varepsilon>0$ and $C>0$ (independent form $L, \beta, r)$ such that, for $|\lambda|<\varepsilon$, we have:

1. For $r>0$ (metallic phase),

$$
\hat{S}_{L, \beta}\left(\mathbf{k} \pm p_{F}\right)=\frac{\left[k_{0}^{2}+\alpha(\lambda)^{2}(\cos k-1+v(\lambda))^{2}\right]^{\eta(\lambda)}}{-i k_{0}+\alpha(\lambda)(\cos k-1+v(\lambda))}\left(1+\lambda R_{S}(\lambda, \mathbf{k})\right)
$$

where

$$
\begin{aligned}
& v(\lambda)=r+\lambda r R_{v}(\lambda) \quad \alpha(\lambda)=1+\lambda R_{\alpha}(\lambda) \\
& \eta(\lambda)=b \lambda^{2} r+\lambda^{3} r^{\frac{3}{2}} R_{\eta}(\lambda)
\end{aligned}
$$

with $b>0$ a constant and $\left|R_{i}\right| \leq C$ for $i=S, v, \alpha$ and $\eta$. 
2. For $r=0$ (critical point)

$$
\hat{S}_{L, \beta}(\mathbf{k})=\frac{1+\lambda R_{S}(\lambda, \mathbf{k})}{-i k_{0}+\alpha(\lambda)(\cos (k)-1)}
$$

where $\alpha(\lambda)=1+\lambda R_{\alpha}(\lambda)$ and $\left|R_{i}\right| \leq C$ for $i=\alpha, S$.

3. For $r<0$ (insulating phase)

$$
\left|\hat{S}_{L, \beta}(\mathbf{k})\right| \leq \frac{C}{|r|}
$$

Clearly, by symmetry, similar results hold at the top of the band by setting $h=1-r$.

From the above result we see that in the metallic phase Luttinger liquid behavior is present; indeed the interaction changes the location of the Fermi points from $p_{F}= \pm \cos ^{-1}(-1+r)$ to $p_{F}=\cos ^{-1}(-1+r+O(\lambda r))$ and, more remarkably, produces an anomalous behavior in the two point Schwinger function due to the presence of the critical exponent $\eta$. Luttinger liquid behavior persists up to the critical point (corresponding to a strong coupling phase in the effective relativistic description); interestingly, the critical exponent becomes smaller the closer one is to the critical point. This is due to the fact that the effective coupling is $O(\lambda r)$ (and not $O(\lambda)$ ), so that the effective coupling divided by the Fermi velocity is $O(\sqrt{r})$ and indeed small for small $r$

At the critical point no anomalous exponent is present; the asymptotic behavior is qualitatively the same as in the non interacting case, up to a finite wave function renormalization and the presence of $\alpha$. Finally in the $r<0$ again an insulating behavior is found, as the 2-point function has no singularities.

The proof of the above result is based on a rigorous implementation of the Wilsonian RG methods. There is a natural momentum scale, which is $O(r)$, separating two different regimes. In the first regime, described in section 2 , the interaction appears to be relevant; however, Pauli principle shows that the relevant contributions are vanishing and the theory turns out to be effectively superrenormalizable: all the interactions are irrelevant and their effect is to produce a finite renormalization of the parameters. In the insulating phase or at the critical point, only this regime is present. In contrast, in the metallic phase there is a second regime, described in section 3, in which the relevant contribution are non vanishing (the presence of the Fermi points introduces an extra label in the fermionic fields). The local quartic terms are therefore marginal and produce the critical exponents; in this second regime, one has to check carefully that the small divisors due to the fact that the Fermi velocity is small are compensated by the small factors coming from the integration of the first regime. 


\section{Renormalization Group integration: the first regime}

\subsection{Grassmann representation}

We introduce a set of anticommuting variables $\psi_{\mathbf{x}}^{ \pm}$such that $\left\{\psi_{\mathbf{x}}^{ \pm}, \psi_{\mathbf{y}}^{ \pm}\right\}=0$ for every $\mathbf{x}, \mathbf{y}$. Given the propagator

$$
g_{M, L, \beta}(\mathbf{x}-\mathbf{y})=\frac{1}{\beta L} \sum_{\mathbf{k} \in \mathscr{D}} e^{i \mathbf{k}(\mathbf{x}-\mathbf{y})} \frac{\chi_{0}\left(\gamma^{-M}\left|k_{0}\right|\right)}{-i k_{0}+\cos k+h}
$$

where $\chi_{0}(t)$ is a smooth even compact support function equal to 1 for $|t| \leq 1$ and equal to 0 for $|t| \geq \gamma$, for some $1<\gamma \leq 2$, we define the Grasmann integration $P(d \psi)$ on the Grassman algebra generated by the $\psi_{\mathbf{x}}^{ \pm}$by setting

$$
\int P(d \psi) \prod_{i=1}^{n} \psi_{\mathbf{x}_{i}}^{-} \prod_{j=1}^{n} \psi_{\mathbf{y}_{j}}^{+}=\operatorname{det} G
$$

where $G$ is the $n \times n$ matrix with entries $G_{i, j}=g_{M, L, \beta}\left(\mathbf{x}_{i}-\mathbf{y}_{j}\right)$. We can extend this definition to a generic monomial in the $\psi^{ \pm}$using the anticommutation rule and to the full algebra by linearity.

Observe that if $\mathbf{x} \neq(0, n \beta)$

$$
\lim _{M \rightarrow \infty} g_{M, L, \beta}(\mathbf{x})=S_{0, L, \beta}(\mathbf{x})
$$

while for $\mathbf{x}=(0, n \beta)$

$$
\lim _{M \rightarrow \infty} g_{M, L, \beta}(\mathbf{x})=\frac{S_{0, L, \beta}\left(0^{+}, 0\right)+S_{0, L, \beta}\left(0^{-}, 0\right)}{2}
$$

while $S_{0, L, \beta}(n \beta, 0)=S_{0, L, \beta}\left(0^{-}, 0\right)$.

By extending the Grassmann algebra with a new set of anticommuting variables $\phi_{\mathbf{x}}^{ \pm}$, we can define the Generating Functional $\mathscr{W}(\phi)$ as the following Grassmann integral

$$
e^{\mathscr{W}_{M}(\phi)}=\int P(d \psi) e^{\mathscr{V}(\psi)+(\psi, \phi)}
$$

where, if $\int d \mathbf{x}=\int d x_{0} \sum_{x}$, we set

$$
\begin{aligned}
& (\psi, \phi)=\int d \mathbf{x}\left[\psi_{\mathbf{x}}^{+} \phi_{\mathbf{x}}^{-}+\psi_{\mathbf{x}}^{-} \phi_{\mathbf{x}}^{+}\right] \\
& \mathscr{V}(\psi)=\lambda \int d \mathbf{x} d \mathbf{y} v(\mathbf{x}-\mathbf{y}) \psi_{\mathbf{x}}^{+} \psi_{\mathbf{x}}^{-} \psi_{\mathbf{y}}^{+} \psi_{\mathbf{y}}^{-}+\bar{v} \int d \mathbf{x} \psi_{\mathbf{x}}^{+} \psi_{\mathbf{x}}^{-}
\end{aligned}
$$


and $v(\mathbf{x}-\mathbf{y})=\delta\left(x_{0}-y_{0}\right) v(x-y) ;$ moreover

$$
\bar{v}=\lambda \hat{v}(0)\left[\frac{S_{0, L, \beta}\left(0,0^{+}\right)-S_{0, L, \beta}\left(0,0^{-}\right)}{2}\right] .
$$

The presence in (19) of the counterterm $\bar{v}$ is necessary to take into account the difference between $g(\mathbf{x})$ and $S_{0}(\mathbf{x})$, see (18).

We define the Schwinger functions as

$$
S_{M, L, \beta}(\mathbf{x}-\mathbf{y})=\left.\frac{\partial^{2}}{\partial \phi_{\mathbf{x}}^{+} \partial \phi_{\mathbf{y}}^{-}} W(\phi)\right|_{\phi=0}
$$

One can easily check, see for instance Proposition 2.1 of [8], that if $S_{\beta, L, M}$ is analytic for $|\lambda| \leq \varepsilon$ then $\lim _{M \rightarrow \infty} S_{M, L, \beta}(\mathbf{x})$ coincides with $S_{L, \beta}(\mathbf{x})$ for $\lambda \leq \varepsilon$. Thus if we can show that $\varepsilon$ does not depend on $L, \beta$ and that the convergence of $S_{M, L, \beta}(\mathbf{x})$ as to $S_{L, \beta}(\mathbf{x})$ is uniform in $L, \beta$, we can study the two-point function of (4) by analyzing the Generating functional (19).

For definiteness, we take $|r| \leq 1 / 2$. The remaining range of $r$ is covered by the results in [4]. The starting point of the analysis is the following decomposition of the propagator

$$
g_{M, L, \beta}(\mathbf{x}-\mathbf{y})=g^{(>0)}(\mathbf{x}-\mathbf{y})+g^{(\leq 0)}(\mathbf{x}-\mathbf{y})
$$

where

$$
g^{(\leq 0)}(\mathbf{x}-\mathbf{y})=\int d \mathbf{k} e^{i \mathbf{k}(\mathbf{x}-\mathbf{y})} \frac{\chi_{0}\left(\gamma^{-M}\left|k_{0}\right|\right) \chi_{\leq 0}(\mathbf{k})}{-i k_{0}+\cos k+h}
$$

where $\int d \mathbf{k}$ stands for $\frac{1}{\beta L} \sum_{\mathbf{k} \in \mathscr{D}}, \chi_{<0}(\mathbf{k})=\chi_{0}\left(a_{0}^{-1} \sqrt{k_{0}^{2}+(\cos k-1+r)^{2}}\right)$, and $g^{(>0)}(\mathbf{x}-\mathbf{y})$ is equal to (25) with $\chi_{\leq 0}(\mathbf{k})$ replaced by $\left(1-\chi_{\leq 0}(\mathbf{k})\right)$. We chose $a_{0}=\gamma^{-1}(1 / 2-r)$ so that, in the support of $\chi_{<0}(\mathbf{k})$ we have $|k| \leq \pi / 6$. This assures that on the domain of $\chi<0$ we have

$$
c|k| \leq|\sin (k)| \leq C|k|
$$

for suitable constant $c$ and $C$.

By using the addition property of Grassmann integrations we can write

$$
e^{\mathscr{W}(\phi)}=\int P\left(d \psi^{(>0)}\right) P\left(d \psi^{(\leq 0)}\right) e^{\mathscr{V}\left(\psi^{(>0)}+\psi^{(\leq 0)}\right)+\left(\psi^{(>0)}+\psi^{(\leq 0)}, \phi\right)} .
$$

After integrating the field $\psi^{(>0)}$ one obtains

$$
e^{\mathscr{W}(\phi)}=e^{-\beta L F_{0}} \int P\left(d \psi^{(\leq 0)}\right) e^{\mathscr{V}^{(0)}\left(\psi^{(\leq 0}, \phi\right)}
$$


It is known, see for instance Lemma 2.2 of [8] for a proof, that $\mathscr{V}^{(0)}\left(\psi^{(\leq 0}, \phi\right)$ is given by

$$
\mathscr{V}^{(0)}(\psi, \phi)=\sum_{n, m \geq 0} \int d \underline{\mathbf{x}} \int d \underline{\mathbf{y}} \prod_{i=1}^{n} \psi_{\mathbf{x}_{i}}^{\varepsilon_{i}} \prod_{j=1}^{m} \phi_{\mathbf{x}_{j}}^{\sigma_{j}} W_{n, m}(\underline{\mathbf{x}}, \underline{\mathbf{y}})
$$

where $\underline{\mathbf{x}}=\left(\mathbf{x}_{1}, \ldots, \mathbf{x}_{n}\right)$ and $\underline{\mathbf{y}}=\left(\mathbf{y}_{1}, \ldots, \mathbf{y}_{m}\right)$ while $\prod_{i=1}^{n} \psi_{\mathbf{x}_{i}}^{\mathcal{E}_{i}}=1$ if $n=0$ and $\prod_{j=1}^{m} \phi_{\mathbf{y}_{j}}^{\sigma_{j}}=1$ if $m=0$; moreover $W_{n, m}(\underline{\mathbf{x}}, \underline{\mathbf{y}})$ are given by convergent power series in $\lambda$ for $\lambda$ small enough and they decay faster than any power in any coordinate difference. Finally, the limit $M \rightarrow \infty$ of $\mathscr{V}^{(0)}(\psi, \phi)$ exists and is reached uniformly in $\beta, L$.

\subsection{The infrared integration}

Thus we are left with the integration over $\psi^{(\leq 0)}$. The heuristic idea to perform this integration is to decompose $\psi_{\mathbf{x}}^{(\leq 0)}$ as

$$
\psi_{\mathbf{x}}^{(\leq 0)}=\sum_{h=0}^{-\infty} \psi_{\mathbf{x}}^{(h)}
$$

where $\psi_{\mathbf{x}}^{(h)}$ depends only on the momenta $\mathbf{k}$ such that $-i k_{0}+\cos k-1+r \simeq \gamma^{h}$. By using repeatedly the addition property for Grasmann integration this decomposition should allow us to integrate recursively over the $\psi^{(h)}$. The index $h$ is called the scale of the field $\psi^{(h)}$. Two different regimes will naturally appear in the analysis, separated by an energy scale depending on $r$. In this section we describe in detail the integration over the first scale and then we give the recursive procedure. To simplify notation we study only the case $\phi=0$. The general case can be obtained easily.

We saw that after the ultraviolet integration we have

$$
e^{\mathscr{W}(0)}=e^{-\beta L F_{0}} \int P\left(d \psi^{(\leq h)}\right) e^{-\mathscr{V}^{(0)}\left(\psi^{(\leq 0)}\right)}
$$

where $\mathscr{V}^{(0)}\left(\psi^{(\leq 0)}\right)$ is the effective potential on scale 0 and can be written has

$$
\mathscr{V}^{(0)}(\psi)=\sum_{n \geq 1} W_{2 n}^{(0)}(\underline{\mathbf{x}}, \underline{\mathbf{y}}) \prod_{i=1}^{n} \psi_{\mathbf{x}_{i}}^{+} \psi_{\mathbf{y}_{i}}^{-}=\sum_{n \geq 1} \mathscr{V}_{2 n}^{(0)}(\psi)
$$

A direct perturbative analysis suggest that to perform the integration (29) we need a renormalized multiscale integration procedure. In particular, the terms with $n=$ 
1,2 are relevant and the terms with $n=3$ are marginal. For this reason we introduce a localization operator acting on the effective potential as

$$
\mathscr{V}^{(0)}=\mathscr{L}_{1} \mathscr{V}^{(0)}+\mathscr{R}_{1} \mathscr{V}^{(0)}
$$

with $\mathscr{R}_{1}=1-\mathscr{L}_{1}$ and $\mathscr{R}_{1}$ is defined in the following way;

1. $\mathscr{R}_{1} \mathscr{V}_{2 n}^{(0)}=\mathscr{V}_{2 n}^{(0)}$ for $n \geq 4$;

2. for $n=3,2$

$$
\begin{gathered}
\mathscr{R}_{1} \mathscr{V}_{4}^{(0)}(\psi)=\int \prod_{i=1}^{4} d \mathbf{x}_{1} W_{4}^{(0)}(\underline{\mathbf{x}}) \psi_{\mathbf{x}_{1}}^{+} D_{\mathbf{x}_{2}, \mathbf{x}_{1}}^{+} \psi_{\mathbf{x}_{3}}^{-} D_{\mathbf{x}_{4}, \mathbf{x}_{3}}^{-} \\
\mathscr{R}_{1} \mathscr{V}_{6}^{(0)}(\psi)=\int \prod_{i=1}^{6} d \mathbf{x}_{i} W_{6}^{(0)}(\underline{\mathbf{x}}) \psi_{\mathbf{x}_{1}}^{+} D_{\mathbf{x}_{2}, \mathbf{x}_{1}}^{+} D_{\mathbf{x}_{3}, \mathbf{x}_{1}}^{+} \psi_{\mathbf{x}_{4}}^{-} D_{\mathbf{x}_{5}, \mathbf{x}_{4}}^{-} D_{\mathbf{x}_{6}, \mathbf{x}_{4}}^{-}
\end{gathered}
$$

where

$$
D_{\mathbf{x}_{2}, \mathbf{x}_{1}}^{\varepsilon}=\psi_{\mathbf{x}_{2}}^{\varepsilon}-\psi_{\mathbf{x}_{1}}^{\varepsilon}=\left(\mathbf{x}_{2}-\mathbf{x}_{1}\right) \int_{0}^{1} d t \partial \psi_{\mathbf{x}_{1,2}^{\prime}(t)}^{\varepsilon}
$$

with $\mathbf{x}_{1,2}^{\prime}(t)=\mathbf{x}_{1}+t\left(\mathbf{x}_{1}-\mathbf{x}_{2}\right)$ will be called an interpolated point

3. For $n=1$

$$
\mathscr{R}_{1} \mathscr{V}_{2}^{(0)}(\psi)=\int d \mathbf{x}_{1} d \mathbf{x}_{2} W_{2}^{(0)}(\underline{\mathbf{x}}) \psi_{\mathbf{x}_{1}}^{+} H_{\mathbf{x}_{1}, \mathbf{x}_{2}}^{-}
$$

where

$$
\begin{aligned}
H_{\mathbf{x}_{1}, \mathbf{x}_{2}}^{-}= & \psi_{\mathbf{x}_{2}}^{-}-\psi_{\mathbf{x}_{1}}^{-}-\left(x_{0,1}-x_{0,2}\right) \partial_{0} \psi_{\mathbf{x}_{1}}^{-}-\left(x_{1}-x_{2}\right) \partial_{1} \psi_{\mathbf{x}_{1}}^{-}- \\
& \frac{1}{2}\left(x_{1}-x_{2}\right)^{2} \widetilde{\Delta}_{1} \psi
\end{aligned}
$$

and

$$
\begin{aligned}
& \widetilde{\partial}_{1} \psi_{\mathbf{x}}^{-}=\frac{1}{2}\left(\psi_{\mathbf{x}+(0,1)}^{-}-\psi_{\mathbf{x}-(0,1)}^{-}\right)=\int d \mathbf{k} \sin k e^{i \mathbf{k x}} \hat{\psi}_{\mathbf{k}}^{-} \\
& \widetilde{\Delta}_{1} \psi_{\mathbf{x}}^{-}=\psi_{\mathbf{x}+(0,1)}^{-}-2 \psi_{\mathbf{x}}^{-}+\psi_{\mathbf{x}-(0,1)}^{-}=2 \int d \mathbf{k}(\cos k-1) e^{i \mathbf{k} \mathbf{x}} \hat{\psi}_{\mathbf{k}}^{-}
\end{aligned}
$$

As a consequence of the above definitions

$$
\begin{aligned}
\mathscr{L}_{1} \mathscr{V}^{(0)}= & \hat{W}_{2}^{(0)}(0) \int d \mathbf{x} \psi_{\mathbf{x}}^{+} \psi_{\mathbf{x}}^{-}+\partial_{0} \hat{W}_{2}^{(0)}(0) \int d \mathbf{x} \psi_{\mathbf{x}}^{+} \partial_{0} \psi_{\mathbf{x}}^{-}+ \\
& \frac{1}{2} \partial_{1}^{2} \hat{W}_{2}^{(0)}(0) \int d \mathbf{x} \psi_{\mathbf{x}}^{+} \partial_{1}^{2} \psi_{\mathbf{x}}^{-}
\end{aligned}
$$

where we have used that 
i. $g^{(0)}\left(k_{0}, k\right)=g^{(0)}\left(k_{0},-k\right)$, so that we get

$$
\partial_{1} \hat{W}_{2}^{(0)}(0)=0
$$

ii. There are no terms in $\mathscr{L}_{1} \mathscr{V}^{(0)}$ with four or six fermionic fields, as

$$
\psi_{\mathbf{x}_{1}}^{\varepsilon} D_{\mathbf{x}_{2}, \mathbf{x}_{1}}^{\varepsilon}=\psi_{\mathbf{x}_{1}}^{\varepsilon} \psi_{\mathbf{x}_{2}}^{\varepsilon}
$$

and therefore $\mathscr{R}_{1} \mathscr{V}_{4}^{(0)}=\mathscr{V}_{4}^{(0)}$ and $\mathscr{R}_{1} \mathscr{V}_{6}^{(0)}=\mathscr{V}_{6}^{(0)}$. As a consequence (32) (33) just represent a different way to write the four and six field contribution to the effective potential. This representation will be useful in the following where we will exploit the dimensional gain due to the zero term $\mathbf{x}_{2}-\mathbf{x}_{1}$ and the derivative in eq. 34.

We will call $\mathscr{L}_{1} \mathscr{V}^{(h)}$ the relevant part of the effective potential. Since it is quadratic in the fields, we can include it in the free integration finding

$$
e^{\mathscr{W}(0)}=e^{-\beta L\left(F_{0}+e_{0}\right)} \int \widetilde{P}\left(d \psi^{(\leq 0)}\right) e^{-\mathscr{R}_{1} \mathscr{V}^{(0)}\left(\psi^{(\leq 0)}\right)}
$$

where the propagator of $\widetilde{P}\left(d \psi^{(\leq 0)}\right)$ is now

$$
\widetilde{g}^{(\leq 0)}(\mathbf{x})=\int d \mathbf{k} e^{i \mathbf{k x}} \frac{\chi_{\leq 0}(\mathbf{k})}{-i k_{0}\left(1+z_{-1}\right)+\left(1+\alpha_{-1}\right)(\cos k-1)+r+\gamma^{-1} \mu_{-1}}
$$

and

$$
\begin{aligned}
& z_{-1}=z_{0}+\chi_{\leq 0}(\mathbf{k}) \partial_{0} \hat{W}_{2}^{(0)}(0) \quad \alpha_{-1}=\alpha_{0}+\chi_{\leq 0}(\mathbf{k}) \partial_{1}^{2} \hat{W}_{2}^{(0)}(0) \\
& \mu_{h-1}=\mu_{0}+\chi_{\leq 0}(\mathbf{k}) \gamma^{-0} \hat{W}_{2}^{(0)}(0)
\end{aligned}
$$

where $z_{0}=\alpha_{0}=\mu_{0}=0$ but we have added them in (42) for later reference.

We can now write

$$
\widetilde{g}^{(\leq 0)}(\mathbf{x})=g^{(\leq-1)}(\mathbf{x})+\widetilde{g}^{(0)}(\mathbf{x})
$$

where

$$
g^{(\leq-1)}(\mathbf{x})=\int d \mathbf{k} e^{i \mathbf{k x}} \frac{\chi_{\leq-1}(\mathbf{k})}{-i k_{0}\left(1+z_{-1}\right)+\left(1+\alpha_{-1}\right)(\cos k-1)+r+\gamma^{-1} \mu_{-1}}
$$

with

$$
\chi_{<-1}(\mathbf{k})=\chi_{0}\left(\gamma a_{0}^{-1} \sqrt{\left(1+z_{-1}\right)^{2} k_{0}^{2}+\left(\left(1+\alpha_{-1}\right) \cos k-1+r+\gamma^{-1} \mu_{-1}\right)^{2}}\right) .
$$


Clearly

$$
\widetilde{g}^{(0)}(\mathbf{x})=\int d \mathbf{k} e^{i \mathbf{k} \mathbf{x}} \frac{f_{0}(\mathbf{k})}{-i k_{0}\left(1+z_{-1}\right)+\left(1+\alpha_{-1}\right)(\cos k-1)+r+\gamma^{-1} \mu_{-1}}
$$

where

$$
f_{0}(\mathbf{k})=\chi_{\leq 0}(\mathbf{k})-\chi_{\leq-1}(\mathbf{k}) .
$$

Using again the addition property for Grassmann integrations we can rewrite (29) and perform the integration over $\psi^{(0)}$ as

$$
\begin{aligned}
e^{\mathscr{W}(0)} & =e^{-\beta L\left(F_{0}+e_{0}\right)} \int P\left(d \psi^{(\leq-1)}\right) \int \widetilde{P}\left(d \psi^{(0)}\right) e^{-\mathscr{R}_{1} \mathscr{V}^{(0)}\left(\psi^{(\leq 0)}\right)}= \\
& =e^{-\beta L F_{-1}} \int P\left(d \psi^{(\leq-1)}\right) e^{-\mathscr{V}^{(-1)}\left(\psi^{(\leq-1)}\right)}
\end{aligned}
$$

where $\widetilde{P}\left(d \psi^{(0)}\right)$ is the integration with propagator $\widetilde{g}^{(0)}(\mathbf{x}), P\left(d \psi^{(\leq 1)}\right)$ is the integration with propagator $\widetilde{g}^{(\leq 1)}(\mathbf{x})$ and

$$
e^{-\beta L e_{0}-\mathscr{V}^{(-1)}\left(\psi^{(\leq-1)}\right)}=\int \widetilde{P}\left(d \psi^{(0)}\right) e^{-\mathscr{R}_{1} \mathscr{V}^{(0)}\left(\psi^{(\leq 0)}\right)}
$$

The fact that this integration is well defined follows from the properties of the propagator $\widetilde{g}^{(0)}(\mathbf{x})$ that will be derived in Lemma 2.1 below.

We can now repeat the above procedure iteratively. At the $h$ step (i.e. at scale h) we start with the integration

$$
e^{\mathscr{W}(0)}=e^{-\beta L F_{h}} \int P\left(d \psi^{(\leq h)}\right) e^{-\mathscr{V}^{(h)}\left(\psi^{(\leq h)}\right)}
$$

defined by the propagator

$$
g^{(\leq h)}(\mathbf{x})=\int d \mathbf{k} e^{i \mathbf{k} \mathbf{x}} \frac{\chi_{\leq h}(\mathbf{k})}{-i k_{0}\left(1+z_{h}\right)+\left(1+\alpha_{h}\right)(\cos k-1)+r+\gamma^{h} \mu_{h}}
$$

with

$$
\chi_{\leq h}(\mathbf{k})=\chi_{0}\left(\gamma^{-h} a_{0}^{-1} \sqrt{\left(1+z_{h}\right)^{2} k_{0}^{2}+\left(\left(1+\alpha_{h}\right)(\cos k-1)+r+\gamma^{h} \mu_{h}\right)^{2}}\right) .
$$

and the effective potential on scale $h$ is given by

$$
\mathscr{V}^{(h)}(\psi)=\sum_{n \geq 1} W_{2 n}^{(h)}(\underline{\mathbf{x}}, \underline{\mathbf{y}}) \prod_{i=1}^{n} \psi_{\mathbf{x}_{i}}^{+} \psi_{\mathbf{y}_{i}}^{-}=\sum_{n \geq 1} V_{2 n}^{(h)}(\psi)
$$


Again we can apply the operator $\mathscr{L}_{1}$ to $\mathscr{V}^{(h)}$ to get

$$
\mathscr{V}^{(h)}=\mathscr{L}_{1} \mathscr{V}^{(h)}+\mathscr{R}_{1} \mathscr{V}^{(h)} .
$$

where $\mathscr{R}$ is defined exactly as in the case of $\mathscr{V}^{(0)}$ and

$$
\begin{aligned}
\mathscr{L}_{1} \mathscr{V}^{(h)}= & \hat{W}_{2}^{(h)}(0) \int d \mathbf{x} \psi_{\mathbf{x}}^{+} \psi_{\mathbf{x}}^{-}+\partial_{0} \hat{W}_{2}^{(h)}(0) \int d \mathbf{x} \psi_{\mathbf{x}}^{+} \partial_{0} \psi_{\mathbf{x}}^{-}+ \\
& \frac{1}{2} \partial_{1}^{2} \hat{W}_{2}^{(h)}(0) \int d \mathbf{x} \psi_{\mathbf{x}}^{+} \partial_{1}^{2} \psi_{\mathbf{x}}^{-}
\end{aligned}
$$

Moving the local part of the effective potential into the integration we get

$$
e^{\mathscr{W}(0)}=e^{-\beta L\left(F_{h}+e_{h}\right)} \int \widetilde{P}\left(d \psi^{(\leq h)}\right) e^{-\mathscr{R} V^{(h)}\left(\psi^{(\leq h)}\right)}
$$

where the propagator of $\widetilde{P}\left(d \psi^{(\leq h)}\right)$ is

$$
\widetilde{g}^{(\leq h)}(\mathbf{x})=\int d \mathbf{k} e^{i \mathbf{k} \mathbf{x}} \frac{\chi_{\leq h}(\mathbf{k})}{-i k_{0}\left(1+z_{h-1}\right)+\left(1+\alpha_{h-1}\right)(\cos k-1)+r+\gamma^{h-1} \mu_{h-1}}
$$

and the running coupling constants are defined recursively by

$$
\begin{aligned}
& z_{h-1}=z_{h}+\chi_{\leq h}(\mathbf{k}) \partial_{0} \hat{W}_{2}^{(h)}(0) \quad \alpha_{h-1}=\alpha_{h}+\chi_{\leq h}(\mathbf{k}) \partial_{1}^{2} \hat{W}_{2}^{(h)}(0) \\
& \mu_{h-1}=\mu_{h}+\chi_{\leq h}(\mathbf{k}) \gamma^{-h} \hat{W}_{2}^{(h)}(0)
\end{aligned}
$$

Finally we can rewrite (40) as

$$
e^{\mathscr{W}(0)}=e^{-\beta L\left(F_{h}+e_{h}\right)} \int P\left(d \psi^{(\leq h-1)}\right) \int \widetilde{P}\left(d \psi^{(h)}\right) e^{-\mathscr{R}_{1} V^{(h)}\left(\psi^{(\leq h)}\right)}
$$

where $\widetilde{P}\left(d \psi^{(h)}\right)$ has now propagator

$$
\begin{aligned}
\widetilde{g}^{(h)}(\mathbf{x})= & \int d \mathbf{k} e^{i \mathbf{k} \mathbf{x}} \frac{f_{h}(\mathbf{k})}{-i k_{0}\left(1+z_{h-1}\right)+\left(1+\alpha_{h-1}\right)(\cos k-1)+r+\gamma^{h^{*}} \mu_{h-1}}= \\
& \int d \mathbf{k} e^{i \mathbf{k} \mathbf{x}} \hat{g}^{(h)}(\mathbf{k})
\end{aligned}
$$

and $f_{h}(\mathbf{k})=\chi_{h}(\mathbf{k})-\chi_{h-1}(\mathbf{k})$; one can perform the integration over $\psi^{(h)}$

$$
e^{-\beta L \bar{e}_{h}-\mathscr{V}^{h-1}}=\int \widetilde{P}\left(d \psi^{(h)}\right) e^{-\mathscr{R}_{1} \mathscr{V}^{(h)}\left(\psi^{(\leq h)}\right)}
$$

obtaining an expression identical to (49) with $h-1$ replacing $h$, so that the procedure can be iterated. 
To show that the above procedure is well defined we need to study the propagator $\widetilde{g}^{(\leq h)}(\mathbf{x})$. We first have to distinguish two range of scales. Do do this we set

$$
h^{*}=\inf \left\{h\left|a_{0} \gamma^{h+1}>\right| r \mid\right\} .
$$

The construction of the theory for $r>0$, is based on the fact that the behavior of the propagator changes significantly when one reaches the scales $h \simeq h^{*}$. To understand this phenomenon, let's, for simplicity sake, neglect the presence of the running constant in the function $\chi_{\leq h}$. We will see in Lemma 2.1 and Lemma 3.1 below that the presence of $\alpha_{h}, z_{h}$ and $\mu_{h}$ will not change the picture. In this situation, it is easy to see that if $h>h^{*}$ then the domain of $f_{h}(\mathbf{k})$ is a ring of width $\gamma^{h}$ that goes around both Fermi points $\left(0, \pm p_{F}\right)$. At this momentum scale the propagator does not distinguish between $p_{F}$ and $-p_{F}$. On the other hand, when $h<h^{*}$ we have

$$
k_{0}^{2}+(\cos k-1+r)^{2}>a_{0}^{2} \gamma^{2 h+1}
$$

in an open neighbor of the $k_{0}$ axis. This means that the domain of $f_{h}(\mathbf{k})$ splits in two rings, one around $p_{F}$ and the other around $-p_{F}$. In this situation it is convenient to write the propagator as a sum of two quasi-particle propagators, each of which depends only on the momenta close to one of the Fermi points.

Here we need precise estimates on $\widetilde{g}^{(h)}$ for $h>h^{*}$ as reported in the following Lemma. The case $h \leq h^{*}$ will be studied in section 3 .

Lemma 2.1. Assume that there exists a constant $K>0$ such that

$$
\left|z_{h}\right|,\left|\alpha_{h}\right|,\left|\mu_{h}\right|<K|\lambda|
$$

for $h \geq h^{*}$. Then for every $N$ and $\lambda$ small enough we have

$$
\left|\partial_{0}^{n_{0}} \widetilde{\partial}_{1}^{n_{1}} \widetilde{\boldsymbol{g}}^{(h)}(\mathbf{x})\right| \leq C_{N} \frac{\gamma^{\frac{h}{2}}}{1+\left[\gamma^{h}\left|x_{0}\right|+\gamma^{\frac{h}{2}}|x|\right]^{N}} \gamma^{h\left(n_{0}+n_{1} / 2\right)}
$$

with $C_{N}$ independent form $K$.

Proof. We start observing that the, in the support of $f_{h}(\mathbf{k})$ we have

$$
\left(1+z_{h}\right)\left|k_{0}\right| \leq \gamma^{h+1} a_{0} \quad\left|\left(1+\alpha_{h}\right)(\cos k-1)+r+\gamma^{h} \mu_{h}\right| \leq \gamma^{h+1} a_{0}
$$

From this we get that

$$
|\sin (k)| \leq 2 \sqrt{1-\cos (k)} \leq 2 \sqrt{\frac{r+K \gamma^{h}|\lambda|+a_{0} \gamma^{h+1}}{1-K|\lambda|}} \leq C \gamma^{\frac{h}{2}}
$$


so that also $|k| \leq C \gamma^{\frac{h}{2}}$. It follows that

$$
\int f_{h}(\mathbf{k}) d \mathbf{k} \leq C \gamma^{\frac{3}{2} h}
$$

To prove the statement for $n_{0}=n_{1}=0$, that we just need to show that

$$
\left|x_{0}^{N_{0}} x^{N_{1}} \widetilde{g}^{(h)}(\mathbf{x})\right| \leq C \gamma^{h\left(\frac{1}{2}-N_{0}-\frac{N_{1}}{2}\right)}
$$

We will use that

$$
x_{0}^{N_{0}} x^{N_{1}} \widetilde{g}^{(h)}(\mathbf{x})=\int d \mathbf{k} e^{i \mathbf{k} \mathbf{x}} \partial_{0}^{N_{0}} \partial_{1}^{N_{1}} \hat{g}^{(h)}(\mathbf{k}) .
$$

To estimate the above derivatives we observe that

$$
\partial_{0}^{N_{0}} \partial_{1}^{N_{1}} \hat{g}^{(h)}(\mathbf{k})=\sum_{P_{1}=1}^{N_{1}} \sum_{\sum_{i} p_{i}=N_{1}-P_{1}} A_{P_{1}, p_{i}} \partial_{0}^{N_{0}} \partial_{\cos (k)}^{P_{1}} \hat{g}^{(h)}(\mathbf{k}) \prod_{i=i}^{P_{1}} \frac{d^{p_{1}}}{d k^{p_{i}}} \sin (k)
$$

where $A_{P_{1}, p_{i}}$ are combinatoric coefficient. It is easy to see that, on the domain of $f_{h}$, we have

$$
\left|\partial_{0}^{N_{0}} \partial_{\cos (k)}^{P_{1}} \hat{g}^{(h)}(\mathbf{k})\right| \leq C \gamma^{-h\left(1+N_{0}+P_{1}\right)} .
$$

If $P_{1} \leq N_{1} / 2$ we can use

$$
\left|\prod_{i=i}^{P_{1}} \frac{d^{p_{1}}}{d k^{p_{i}}} \sin (k)\right| \leq 1 \quad \text { and } \quad \gamma^{-h\left(1+N_{0}+P_{1}\right)} \leq \gamma^{-h\left(1+N_{0}+\frac{N_{1}}{2}\right)}
$$

while, if $P_{1}>N_{1} / 2$, at least $2 P_{1}-N_{1}$ of the $p_{i}$ in the above product must be zero so that

$$
\begin{aligned}
\left|\prod_{i=i}^{P_{1}} \frac{d^{p_{1}}}{d k^{p_{i}}} \sin (k)\right| & \leq C \gamma^{\left(2 P_{1}-N_{1}\right) h} \\
\gamma^{-h\left(1+N_{0}+P_{1}\right)} \gamma^{h\left(2 P_{1}-N_{1}\right)} & \leq \gamma^{-h\left(1+N_{0}+\frac{N_{1}}{2}\right)}
\end{aligned}
$$

In both cases we get

$$
\left|\partial_{0}^{N_{0}} \partial_{1}^{N_{1}} \hat{g}^{(h)}(\mathbf{k})\right| \leq C \gamma^{h\left(1+N_{0}+\frac{N_{1}}{2}\right)} .
$$

Combining with (64) we get (65). Observe now that

$$
\partial_{0}^{n_{0}} \partial_{1}^{n_{1}} \widetilde{g}^{(h)}(\mathbf{x})=(i)^{n_{0}+n_{1}} \int d \mathbf{k} e^{i \mathbf{k x}} k_{0}^{n_{0}} \sin ^{n_{1}} k \hat{g}^{(h)}(\mathbf{k}) .
$$

The Lemma follows easily reasoning as above and using (62) for the extra powers of $k_{0}$ and $k$. 


\subsection{Tree expansion for the effective potentials.}

The effective potential $V^{(h)}\left(\psi^{(\leq h)}\right)$ can be written in terms of a tree expansion, see [9],[10], defined as follows.

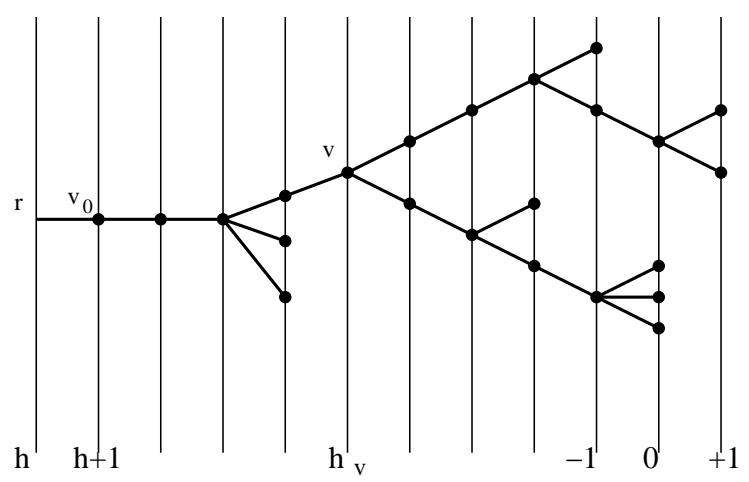

Figure 1: A tree $\tau \in \mathscr{T}_{h, n}$ with its scale labels.

1. On the plane, we draw the vertical lines at horizontal position given by the integers from $h$ to 1 , see Fig. 1. We select one point on the line at $h$ (the root) and one point on the line at $h+1$ (the first vertex $v_{0}$ ). On the line at $k$, with $h+1<k \leq 1$, we select $m_{k}>0$ points (the vertex at scale $k$ ). We call $M_{k}$ the set of vertex at scale $k$. To each vertex $v$ in $M_{k}$ we associate exactly one vertex $v^{\prime}$ in $M_{k-1}$ and we draw a line between these two vertices. The vertex $v^{\prime}$ is called the predecessor of $v$. Finally we require that if $v$ and $w$ are in $M_{k}$ with $v$ below $w$ then $v^{\prime}$ is below or equal to $w^{\prime}$. The final results of this procedure is clearly a tree with root $r$.

2. Given a vertex $v$ on scale $k$, let $s_{v}$ be the number of vertex on scale $h+1$ linked to $v$. If $s_{v}=0$ we say that $v$ is a end point. The number $n$ of endpoint is called the order of the tree. If $s_{v}=1$ we say that $v$ is a trivial vertex. Finally if $s_{v}>1$ we say that $v$ is a branching point or non-trivial vertex. The tree structure induce a natural ordering (denoted by $<$ ) on the vertex such that if $v_{1}$ and $v_{2}$ are two vertices and $v_{1}<v_{2}$, then $h_{v_{1}}<h_{v_{2}}$. We call $\mathscr{T}_{h, n}$ the set of all tree constructed in this way.

3. Given a vertex $v$ of $\tau \in \mathscr{T}_{h, n}$ that is not an endpoint, we can consider the subtrees of $\tau$ with root $v$, which correspond to the connected components of the restriction of $\tau$ to the vertices $w>v$. If a subtree with root $v$ contains only $v$ and an endpoint on scale $h_{v}+1$, we will call it a trivial subtree. 
4. With each endpoint $v$ we associate one of the monomials contributing to $\mathscr{R}_{1} \mathscr{V}^{(0)}\left(\psi^{\left(\leq h_{v}-1\right)}\right)$ and a set $\mathbf{x}_{v}$ of space-time points.

5. We introduce a field label $f$ to distinguish the field variables appearing in the terms associated with the endpoints described in item 4); the set of field labels associated with the endpoint $v$ will be called $I_{v}, \mathbf{x}(f), \varepsilon(f)$ will be the position and type of the field variable $f$. Observe that $\left|I_{v}\right|$ is the order of the monomial contributing to $\mathscr{V}^{(0)}\left(\psi^{\left(\leq h_{v}-1\right)}\right)$ and associated to $v$. Analogously, if $v$ is not an endpoint, we shall call $I_{v}$ the set of field labels associated with the endpoints following the vertex $v$; finally we will call the set of point $\mathbf{x}(f)$ for $f \in I_{v}$ the cluster associated to $v$.

Given $\mathscr{U}_{i}\left(\psi^{(h)}\right)$ for $i=1, \ldots, n$ we define the truncated expectation on scale $h$ as

$$
\begin{aligned}
\mathscr{E}_{h} T & {\left[\mathscr{U}_{1}\left(\psi^{(h)}\right) ; \ldots ; \mathscr{U}_{n}^{(h)}\left(\psi^{(h)}\right)\right]=} \\
& \left.\frac{\partial^{n}}{\partial \lambda_{1} \cdots \partial \lambda_{n}} \log \int P\left(d \psi^{(h)}\right) e^{\lambda_{1} \mathscr{U}_{1}\left(\psi^{(h)}\right)+\cdots+\lambda_{n} \mathscr{U}_{n}\left(\psi^{(h)}\right)}\right|_{\lambda_{1}=\ldots=\lambda_{N}=0}
\end{aligned}
$$

In terms of above trees, the effective potential $\mathscr{V}^{(h)}, h \leq-1$, can be written as

$$
\mathscr{V}^{(h)}\left(\psi^{(\leq h)}\right)+\beta L \bar{e}_{h+1}=\sum_{n=1}^{\infty} \sum_{\tau \in \mathscr{T}_{h, n}} \mathscr{V}^{(h)}\left(\tau, \psi^{(\leq h)}\right),
$$

where, if $v_{0}$ is the first vertex of $\tau$ and $\tau_{1}, \ldots, \tau_{s}\left(s=s_{\nu_{0}}\right)$ are the subtrees of $\tau$ with root $v_{0}, \mathscr{V}^{(h)}\left(\tau, \psi^{(\leq h)}\right)$ is defined inductively as follows:

$\mathrm{i}$ if $s>1$, then

$$
\begin{aligned}
& \mathscr{V}^{(h)}\left(\tau, \psi^{(\leq h)}\right)= \\
& \quad \frac{(-1)^{s+1}}{s !} \mathscr{E}_{h+1}^{T}\left[\overline{\mathscr{V}}^{(h+1)}\left(\tau_{1}, \psi^{(\leq h+1)}\right) ; \ldots ; \overline{\mathscr{V}}^{(h+1)}\left(\tau_{s}, \psi^{(\leq h+1)}\right)\right]
\end{aligned}
$$

where $\overline{\mathscr{V}}^{(h+1)}\left(\tau_{i}, \psi^{(\leq h+1)}\right)$ is equal to $\mathscr{R}_{1} \mathscr{V}^{(h+1)}\left(\tau_{i}, \psi^{(\leq h+1)}\right)$ if the subtree $\tau_{i}$ contains more than one end-point, or if it contains one end-point but it is not a trivial subtree; it is equal to $\mathscr{R}_{1} \mathscr{V}^{(0)}\left(\tau_{i}, \psi^{(\leq h+1)}\right)$ if $\tau_{i}$ is a trivial subtree;

ii if $s=1$ and $\tau_{1}$ is not a trivial subtree, then $\mathscr{V}^{(h)}\left(\tau, \psi^{(\leq h)}\right)$ is equal to

$$
\mathscr{E}_{h+1}^{T}\left[\mathscr{R}_{1} \mathscr{V}^{(h+1)}\left(\tau_{1}, \psi^{(\leq h+1)}\right)\right] .
$$


Using its inductive definition, the right hand side of (74) can be further expanded, and in order to describe the resulting expansion we need some more definitions.

We associate with any vertex $v$ of the tree a subset $P_{v}$ of $I_{v}$, the external fields of $v$. These subsets must satisfy various constraints. First of all, if $v$ is not an endpoint and $v_{1}, \ldots, v_{s_{v}}$ are the $s_{v} \geq 1$ vertices immediately following it, then $P_{v} \subseteq \cup_{i} P_{v_{i}}$; if $v$ is an endpoint, $P_{v}=I_{v}$. If $v$ is not an endpoint, we shall denote by $Q_{v_{i}}$ the intersection of $P_{v}$ and $P_{v_{i}}$; this definition implies that $P_{v}=\cup_{i} Q_{v_{i}}$. The union $\mathscr{I}_{v}$ of the subsets $P_{v_{i}} \backslash Q_{v_{i}}$ is, by definition, the set of the internal fields of $v$, and is non empty if $s_{v}>1$. Given $\tau \in \mathscr{T}_{h, n}$, there are many possible choices of the subsets $P_{v}$, $v \in \tau$, compatible with all the constraints. We shall denote $\mathscr{P}_{\tau}$ the family of all these choices and $\mathbf{P}$ the elements of $\mathscr{P}_{\tau}$.

With these definitions, we can rewrite $\mathscr{V}^{(h)}\left(\tau, \psi^{(\leq h)}\right)$ in the r.h.s. of (74) as:

$$
\begin{aligned}
& \mathscr{V}^{(h)}\left(\tau, \psi^{(\leq h)}\right)=\sum_{\mathbf{P} \in \mathscr{P}_{\tau}} \mathscr{V}^{(h)}(\tau, \mathbf{P}), \\
& \mathscr{V}^{(h)}(\tau, \mathbf{P})=\int d \mathbf{x}_{v_{0}} \widetilde{\psi}^{(\leq h)}\left(P_{v_{0}}\right) K_{\tau, \mathbf{P}}^{(h+1)}\left(\mathbf{x}_{v_{0}}\right),
\end{aligned}
$$

where

$$
\widetilde{\psi}^{(\leq h)}\left(P_{v}\right)=\prod_{f \in P_{v}} \psi_{\mathbf{x}(f)}^{(\leq h) \varepsilon(f)}
$$

and $K_{\tau, \mathbf{P}}^{(h+1)}\left(\mathbf{x}_{v_{0}}\right)$ is defined inductively by the equation, valid for any $v \in \tau$ which is not an endpoint,

$$
K_{\tau, \mathbf{P}}^{\left(h_{v}\right)}\left(\mathbf{x}_{v}\right)=\frac{1}{s_{v} !} \prod_{i=1}^{s_{v}}\left[K_{v_{i}}^{\left(h_{v}+1\right)}\left(\mathbf{x}_{v_{i}}\right)\right] \mathscr{E} \mathscr{E}_{h_{v}} T\left[\widetilde{\psi}^{\left(h_{v}\right)}\left(P_{v_{1}} \backslash Q_{v_{1}}\right), \ldots, \widetilde{\psi}^{\left(h_{v}\right)}\left(P_{v_{s_{v}}} \backslash Q_{v_{s_{v}}}\right)\right]
$$

where $\widetilde{\psi}^{\left(h_{v}\right)}\left(P_{v_{i}} \backslash Q_{v_{i}}\right)$ has a definition similar to (77). Moreover, if $v_{i}$ is an endpoint $K_{v_{i}}^{\left(h_{v}+1\right)}\left(\mathbf{x}_{v_{i}}\right)$ is equal to one of the kernels of the monomials contributing to $\mathscr{R}_{1} \mathscr{V}^{(0)}\left(\psi^{\left(\leq h_{v}\right)}\right)$; if $v_{i}$ is not an endpoint, $K_{v_{i}}^{\left(h_{v}+1\right)}=K_{\tau_{i}, \mathbf{P}_{i}}^{\left(h_{v}+1\right)}$, where $\mathbf{P}_{i}=\left\{P_{w}, w \in\right.$ $\left.\tau_{i}\right\}$.

The final form of our expansions not yet given by (74)-(78). We can further decompose $\mathscr{V}^{(h)}(\tau, \mathbf{P})$, by using the following representation of the truncated expectation in the r.h.s. of (78). Let us put $s=s_{v}, P_{i} \equiv P_{v_{i}} \backslash Q_{v_{i}}$; moreover we order in an arbitrary way the sets $P_{i}^{ \pm} \equiv\left\{f \in P_{i}, \varepsilon(f)= \pm\right\}$, we call $f_{i j}^{ \pm}$their elements and we define $\mathbf{x}^{(i)}=\cup_{f \in P_{i}^{-}} \mathbf{x}(f), \mathbf{y}^{(i)}=\cup_{f \in P_{i}^{+}} \mathbf{x}(f), \mathbf{x}_{i j}=\mathbf{x}\left(f_{i j}^{-}\right), \mathbf{y}_{i j}=\mathbf{x}\left(f_{i j}^{+}\right)$. Note that $\sum_{i=1}^{s}\left|P_{i}^{-}\right|=\sum_{i=1}^{s}\left|P_{i}^{+}\right| \equiv n$, otherwise the truncated expectation vanishes.

Then, we use the Brydges-Battle-Federbush [11, 12, 13] formula saying that, up to a sign, if $s>1$,

$$
\mathscr{E}_{h}^{T}\left(\widetilde{\psi}^{(h)}\left(P_{1}\right), \ldots, \widetilde{\psi}^{(h)}\left(P_{s}\right)\right)=\sum_{T} \prod_{l \in T} g^{(h)}\left(\mathbf{x}_{l}-\mathbf{y}_{l}\right) \int d P_{T}(\mathbf{t}) \operatorname{det} G^{h, T}(\mathbf{t}),
$$


where $T$ is a set of lines forming an anchored tree graph between the clusters associated with $v_{i}$ that is $T$ is a set of lines, which becomes a tree graph if one identifies all the points in the same cluster. Moreover $\mathbf{t}=\left\{t_{i i^{\prime}} \in[0,1], 1 \leq i, i^{\prime} \leq s\right\}$, $d P_{T}(\mathbf{t})$ is a probability measure with support on a set of $\mathbf{t}$ such that $t_{i i^{\prime}}=\mathbf{u}_{i} \cdot \mathbf{u}_{i^{\prime}}$ for some family of vectors $\mathbf{u}_{i} \in \mathbb{R}^{s}$ of unit norm. Finally $G^{h, T}(\mathbf{t})$ is a $(n-s+1) \times(n-$ $s+1)$ matrix, whose elements are given by

$$
G_{i j, i^{\prime} j^{\prime}}^{h, T}=t_{i i^{\prime}} g^{(h)}\left(\mathbf{x}_{i j}-\mathbf{y}_{i^{\prime} j^{\prime}}\right),
$$

with $\left(f_{i j}^{-}, f_{i^{\prime} j^{\prime}}^{+}\right)$not belonging to $T$. In the following we shall use (77) even for $s=1$, when $T$ is empty, by interpreting the r.h.s. as equal to 1 , if $\left|P_{1}\right|=0$, otherwise as equal to $\operatorname{det} G^{h}=\mathscr{E}_{h}^{T}\left(\widetilde{\psi}^{(h)}\left(P_{1}\right)\right)$. It is crucial to note that $G^{h, T}$ is a Gram matrix, i.e., the matrix elements in $(80)$ can be written in terms of scalar products:

$$
\begin{aligned}
& t_{i i^{\prime}}{ }^{(h)}\left(\mathbf{x}_{i j}-\mathbf{y}_{i^{\prime} j^{\prime}}\right)= \\
& \quad=\left(\mathbf{u}_{i} \otimes A\left(\mathbf{x}_{i j}-\cdot\right), \mathbf{u}_{i^{\prime}} \otimes B\left(\mathbf{x}_{i^{\prime} j^{\prime}}-\cdot\right)\right) \equiv\left(\mathbf{f}_{\alpha}, \mathbf{g}_{\beta}\right),
\end{aligned}
$$

where

$$
\begin{aligned}
& A(\mathbf{x})=\int d \mathbf{k} e^{-i \mathbf{k x}} \sqrt{f_{h}(\mathbf{k})} \frac{\sqrt{\left|\hat{D}_{h}(\mathbf{k})\right|}}{\hat{D}_{h}(\mathbf{k})}, \\
& B(\mathbf{x})=\int d \mathbf{k} e^{-i \mathbf{k x}} \sqrt{f_{h}(\mathbf{k})} \frac{1}{\sqrt{\left|\hat{D}_{h}(\mathbf{k})\right|}} .
\end{aligned}
$$

where $\hat{D}_{h}(\mathbf{k})=-i k_{0}\left(1+z_{h}\right)+\left(1+\alpha_{h}\right) \cos k-1+r+\gamma^{h} \mu_{h}$. The symbol $(\cdot, \cdot)$ denotes the inner product, i.e.,

$$
\left(\mathbf{u}_{i} \otimes A(\mathbf{x}-\cdot), \mathbf{u}_{i^{\prime}} \otimes B\left(\mathbf{x}^{\prime}-\cdot\right)\right)=\left(\mathbf{u}_{i} \cdot \mathbf{u}_{i^{\prime}}\right) \cdot \int d \mathbf{z} A^{*}(\mathbf{x}-\mathbf{z}) B\left(\mathbf{x}^{\prime}-\mathbf{z}\right),
$$

and the vectors $\mathbf{f}_{\alpha}, \mathbf{g}_{\beta}$ with $\alpha, \beta=1, \ldots, n-s+1$ are implicitly defined by (81). The usefulness of the representation (81) is that, by the Gram-Hadamard inequality, $\left|\operatorname{det}\left(\mathbf{f}_{\alpha}, \mathbf{g}_{\beta}\right)\right| \leq \prod_{\alpha}\left\|\mathbf{f}_{\alpha}\right\|\left\|\mathbf{g}_{\alpha}\right\|$. In our case, $\left\|\mathbf{f}_{\alpha}\right\|,\left\|\mathbf{g}_{\alpha}\right\| \leq C \gamma^{h / 4}$ as it easily follows along the line of the proof of Lemma 2.1. Therefore, $\left\|\mathbf{f}_{\alpha}\right\|\left\|\mathbf{g}_{\alpha}\right\| \leq C \gamma^{\frac{h}{2}}$, uniformly in $\alpha$, so that the Gram determinant can be bounded by $C^{n-s+1} \gamma^{\frac{h}{2}(n-s+1)}$.

If we apply the expansion (79) in each vertex of $\tau$ different from the endpoints, we get an expression of the form

$$
\mathscr{V}^{(h)}(\tau, \mathbf{P})=\sum_{T \in \mathbf{T}} \int d \mathbf{x}_{v_{0}} \widetilde{\psi}^{(\leq h)}\left(P_{v_{0}}\right) W_{\tau, \mathbf{P}, T}^{(h)}\left(\mathbf{x}_{v_{0}}\right) \equiv \sum_{T \in \mathbf{T}} \mathscr{V}^{(h)}(\tau, \mathbf{P}, T),
$$


where $\mathbf{T}$ is a special family of graphs on the set of points $\mathbf{x}_{v_{0}}$, obtained by putting together an anchored tree graph $T_{v}$ for each non trivial vertex $v$. Note that any graph $T \in \mathbf{T}$ becomes a tree graph on $\mathbf{x}_{v_{0}}$, if one identifies all the points in the sets $\mathbf{x}_{v}$, with $v$ an endpoint.

\subsection{Analyticity of the effective potentials.}

Our next goal is the proof of the following result.

Lemma 2.2. There exists a constants $\lambda_{0}>0$, independent of $\beta, L$ and $r$, such that the kernels $W_{l}^{(h)}$ in the domain $|\lambda| \leq \lambda_{0}$, are analytic function of $\lambda$ and satisfy for $h \geq h^{*}$

$$
\frac{1}{\beta L} \int d \mathbf{x}_{1} \cdots d \mathbf{x}_{l}\left|W_{l}^{(h)}\left(\mathbf{x}_{1}, \ldots, \mathbf{x}_{l}\right)\right| \leq \gamma^{h\left(\frac{3}{2}-\frac{l}{4}\right)} \gamma^{\vartheta}(C|\lambda|)^{\max (1, l-1)}
$$

with $\vartheta=\frac{1}{4}$.

Proof. The proof is done by induction. We assume that for $k \geq h+1$ (85) holds together with

$$
\int d \mathbf{x}\left(\left|x_{0}\right|+\left|x_{1}\right|^{2}\right)\left|W_{2}^{(k)}(\mathbf{x})\right| \leq C|\lambda| \gamma^{\vartheta k}
$$

and

$$
\int d \mathbf{x}\left|W_{2}^{(k)}(\mathbf{x})\right| \leq C|\lambda||r| \gamma^{\vartheta k}
$$

The validity of (86) and (87) implies (61).

We now prove that the validity of (85), (86) and (87). Using the tree expansion described above and, in particular, (74), (76), (84), we find that the 1.h.s. of (85) can be bounded above by

$$
\begin{aligned}
& \sum_{n \geq 1} \sum_{\tau \in \mathscr{T}_{h, n}} \sum_{\mathbf{P} \in \mathscr{P}_{\tau}} \sum_{T \in \mathbf{T}} C^{n}\left[\prod_{P_{v_{0}} \mid=l}^{n} C^{p_{i}}|\lambda|^{\frac{p_{i}}{2}-1}\right]\left[\prod_{v=1} \frac{1}{s^{\prime} !} \gamma^{\left.h_{v}\left(\sum_{i=1}^{s v} \frac{\left|P_{v_{i}}\right|}{4}-\frac{\left|P_{v}\right|}{4}-\frac{3}{2}\left(s_{v}-1\right)\right)\right]}\right. \\
& {\left[\prod_{v \text { not e.p. }} \gamma^{-\left(h_{v}-h_{v^{\prime}}\right) z_{1}\left(P_{v}\right)}\right]\left[\prod_{v \text { e.p., }\left|I_{v}\right|=4,6} \gamma^{h_{v^{\prime}}}\right] }
\end{aligned}
$$

where $z_{1}\left(P_{v}\right)=2$ for $\left|P_{v}\right|=6, z_{1}\left(P_{v}\right)=1$ for $\left|P_{v}\right|=4$ and $z_{1}\left(P_{v}\right)=\frac{3}{2}$ for $\left|P_{v}\right|=2$. Note the role of the $\mathscr{R}_{1}$ operation in the above bound; if we neglect $\mathscr{R}_{1}$ we can get 
a similar bound where the second line of eq. $(88)$ is simply replaced by 1 . Its proofs is an immediate consequence of the Gram-Hadamard inequality

$$
\left|\operatorname{det} G^{h_{v}, T_{v}}\left(\mathbf{t}_{v}\right)\right| \leq c^{\sum_{i=1}^{s_{v}}\left|P_{v_{i}}\right|-\left|P_{v}\right|-2\left(s_{v}-1\right)} \cdot \gamma^{\frac{h_{v}}{2}\left(\sum_{i=1}^{s_{v}} \frac{\left|P_{v_{i}}\right|}{2}-\frac{\left|P_{v}\right|}{2}-\left(s_{v}-1\right)\right) .}
$$

and of the decay properties of $g^{(h)}(\mathbf{x})$, implying

$$
\prod_{v \text { not e.p. }} \frac{1}{s_{v} !} \int \prod_{l \in T_{v}} d\left(\mathbf{x}_{l}-\mathbf{y}_{l}\right)\left\|g^{\left(h_{v}\right)}\left(\mathbf{x}_{l}-\mathbf{y}_{l}\right)\right\| \leq c^{n} \prod_{v \text { not e.p. }} \frac{1}{s_{v} !} \gamma^{-h_{v}\left(s_{v}-1\right)} .
$$

If we take into account the subtraction to the 2 field terms and rewriting of the 4 and 6 fields terms involved in the $\mathscr{R}_{1}$ operation we obtain the extra factor

$$
\left[\prod_{v \text { not e.p. }} \gamma^{-\left(h_{v}-h_{v^{\prime}}\right) z_{1}\left(P_{v}\right)}\right]\left[\prod_{v \text { e.p., }\left|I_{v}\right|=4,6} \gamma^{h_{v^{\prime}} \frac{\left|I_{v}\right|-2}{2}}\right]\left[\prod_{v e . p .,\left|I_{v}\right|=2} \gamma^{\frac{3 h_{v^{\prime}}}{2}}\right]
$$

which is produced by the extra zeros and derivatives in the fields $D_{\mathbf{x}_{i}, \mathbf{x}_{j}}$ (when written as in the last of (34) and $H_{\mathbf{x}_{1}, \mathbf{x}_{2}}$; each time or space derivative produce a gain $\gamma^{h_{v^{\prime}}}$ or $\gamma^{h_{v^{\prime}} / 2}$ respectively while the zeros can be associated to the propagators in the anchored tree $T$ (for vertices that are not end points) or to the kernels in $V^{(0)}$ (for the end points) producing a loss bounded by $\gamma^{-h_{v}}$ or $\gamma^{-h_{v} / 2}$. While the origin of such factors can be easily understood by the above dimensional considerations, some care has to be taken to obtain such gains, related to the presence of the interpolated points and to avoid "bad" extra factorials; we refer for instance to section 3 of [4] where a similar bound in an analogous case is derived with all details.

Once the bound (88) is obtained, we have to see if we can sum over the scales and the trees. Let us define $n(v)=\sum_{i: v_{i}^{*}>v} 1$ as the number of endpoints following $v$ on $\tau$. Recalling that $\left|I_{v}\right|$ is the number of field labels associated to the endpoints following $v$ on $\tau$ and using that

$$
\begin{aligned}
& \sum_{v \text { not e.p. }}\left[\sum_{i=1}^{s_{v}}\left|P_{v_{i}}\right|-\left|P_{v}\right|\right]=\left|I_{v_{0}}\right|-\left|P_{v_{0}}\right|, \\
& \sum_{v \text { not e.p. }}\left(s_{v}-1\right)=n-1 \\
& \sum_{v \text { not e.p. }}\left(h_{v}-h\right)\left[\sum_{i=1}^{s_{v}}\left|P_{v_{i}}\right|-\left|P_{v}\right|\right]=\sum_{v \text { not e.p. }}\left(h_{v}-h_{v^{\prime}}\right)\left(\left|I_{v}\right|-\left|P_{v}\right|\right), \\
& \sum_{v \text { not e.p. }}\left(h_{v}-h\right)\left(s_{v}-1\right)=\sum_{v \text { not e.p. }}\left(h_{v}-h_{v^{\prime}}\right)(n(v)-1),
\end{aligned}
$$


we find that $(88)$ can be bounded above by

$$
\begin{aligned}
& \sum_{n \geq 1} \sum_{\tau \in \mathscr{T}, n} \sum_{\substack{\mathbf{P} \in \mathscr{P}_{\tau} \\
\left|P_{v_{0}}\right|=2 l}} \sum_{T \in \mathbf{T}} C^{n} \gamma^{h\left(\frac{3}{2}-\frac{1}{4}\left|P_{v_{0}}\right|+\frac{1}{4}\left|I_{v_{0}}\right|-\frac{3}{2} n\right)}\left[\prod_{i=1}^{n} C^{p_{i}}|\lambda|^{\frac{p_{i}}{2}-1}\right] \\
& {\left[\prod_{v \text { not e.p. }} \frac{1}{s_{v} !} \gamma^{\left(h_{v}-h_{v^{\prime}}\right)\left(\frac{3}{2}-\frac{\left|P_{v}\right|}{4}+\frac{\left|v_{v}\right|}{4}-\frac{3}{2} n(v)+z_{1}\left(P_{v}\right)\right)}\right]} \\
& {\left[\prod_{v \text { e.p. },\left|I_{v}\right|=4,6} \gamma^{h_{v^{\prime}} \frac{\left|I_{v}\right|-2}{2}}\right]\left[\prod_{v \text { e.p., }, I_{v} \mid=2} \gamma^{\frac{3 h_{v^{\prime}}}{2}}\right]}
\end{aligned}
$$

Using the identities

$$
\begin{gathered}
\gamma^{h n} \prod_{v \text { not e.p. }} \gamma^{\left(h_{v}-h_{v^{\prime}}\right) n(v)}=\prod_{v \text { e.p. }} \gamma^{h_{v^{\prime}}}, \\
\gamma^{h\left|I_{v_{0}}\right|} \prod_{v \text { not e.p. }} \gamma^{\left(h_{v}-h_{v^{\prime}}\right)\left|I_{v}\right|}=\prod_{v \text { e.p. }} \gamma^{h_{v^{\prime}}\left|I_{v}\right|},
\end{gathered}
$$

we obtain

$$
\begin{aligned}
& \frac{1}{\beta L} \int d \mathbf{x}_{1} \cdots d \mathbf{x}_{l}\left|W_{l}^{(h)}\left(\mathbf{x}_{1}, \ldots, \mathbf{x}_{l}\right)\right| \leq \\
& \sum_{n \geq 1} \sum_{\tau \in \mathscr{T}, n} \sum_{\substack{\mathbf{P} \in \mathscr{P} \\
\left|P_{v_{0}}\right|=2 l}} \sum_{T \in \mathbf{T}} C^{n} \gamma^{h\left(\frac{3}{2}-\frac{l}{4}\right)}\left[\prod_{i=1}^{n} C^{p_{i}}|\lambda|^{\frac{p_{i}}{2}-1}\right] \\
& \cdot\left[\prod_{v \text { not e.p. }} \frac{1}{s_{v} !} \gamma^{-\left(h_{v}-h_{v^{\prime}}\right)\left(\frac{\left|P_{v}\right|}{4}-\frac{3}{2}+z_{1}\left(P_{v}\right)\right)}\right] \text {. } \\
& \cdot\left[\prod_{v \text { e.p. },\left|I_{v}\right|>6} \gamma^{h_{v^{\prime}}\left(\frac{\left|I_{v}\right|}{4}-\frac{3}{2}\right)}\right]\left[\prod_{v \text { e.p. },\left|I_{v}\right|=2} \gamma^{\frac{h_{v^{\prime}}}{2}}\right]\left[\prod_{v \text { e.p. },\left|I_{v}\right|=4,6} \gamma^{h_{v^{\prime}}}\right]
\end{aligned}
$$

Note that,

$$
\left[\prod_{v \text { e.p. },\left|I_{v}\right|>6} \gamma^{h_{v^{\prime}}\left(\frac{\left|I_{v}\right|}{4}-\frac{3}{2}\right)}\right]\left[\prod_{v \text { e.p., }\left|I_{v}\right|=2} \gamma^{\frac{h_{v^{\prime}}}{2}}\right]\left[\prod_{v \text { e.p., }, I_{v} \mid=4,6} \gamma^{h_{v^{\prime}} \frac{3\left|I_{v}\right|-10}{4}}\right] \leq \gamma^{\frac{\bar{h}}{2}}
$$

with $\bar{h}$ the highest scale label of the tree. Since

$$
\frac{\left|P_{v}\right|}{4}-\frac{3}{2}+z_{1}\left(P_{v}\right) \geq \frac{1}{2}
$$


we see that

$$
\begin{aligned}
& {\left[\prod_{v \text { not e.p. }} \frac{1}{s_{v} !} \gamma^{-\left(h_{v}-h_{v^{\prime}}\right)\left(\frac{\left|P_{v}\right|}{4}-\frac{3}{2}+z_{1}\left(P_{v}\right)\right)}\right] \gamma^{\frac{\bar{h}}{2}} \leq} \\
& {\left[\prod_{v \text { not e.p. }} \frac{1}{s_{v} !} \gamma^{-\left(h_{v}-h_{v^{\prime}}\right) \eta\left(\frac{\left|P_{v}\right|}{4}-\frac{3}{2}+z_{1}\left(P_{v}\right)\right)}\right] \gamma^{h \frac{(1-\eta)}{2}} .}
\end{aligned}
$$

for any $0<\eta<1$. On the other hand we have that

$$
\frac{\left|P_{v}\right|}{4}-\frac{3}{2}+z_{1}\left(P_{v}\right) \geq \frac{\left|P_{v}\right|}{16}
$$

so that, using also eq.967, we get

$$
\prod_{v \text { not e.p. }} \frac{1}{s_{v} !} \gamma^{-\left(h_{v}-h_{v^{\prime}}\right) \eta\left(\frac{\left|P_{v}\right|}{4}-\frac{3}{2}+z_{1}\left(P_{v}\right)\right)} \leq\left[\prod_{v \text { not e.p. }} \frac{1}{s_{v} !} \gamma^{-\frac{\eta}{4}\left(h_{v}-h_{v^{\prime}}\right)}\right]\left[\prod_{v \text { not e.p. }} \gamma^{-\frac{\eta}{32}\left|P_{v}\right|}\right]
$$

Collecting the above estimates and using that the number of terms in $\sum_{T \in \mathbf{T}}$ is bounded by $C^{n} \prod_{v}$ not e.p. $s_{v}$ ! we obtain

$$
\begin{gathered}
\frac{1}{\beta L} \int d \mathbf{x}_{1} \cdots d \mathbf{x}_{l}\left|W_{l}^{(h)}\left(\mathbf{x}_{1}, \ldots, \mathbf{x}_{l}\right)\right| \leq \gamma^{h\left(\frac{3}{2}-\frac{l}{4}\right)} \gamma^{\frac{1-\eta}{2}} h \sum_{n \geq 1} C^{n} \sum_{\tau \in \mathscr{T}, n}\left[\prod_{i=1}^{n} C^{p_{i}}|\lambda|^{\frac{p_{i}}{2}-1}\right] \\
\cdot\left[\prod_{v \text { not e.p. }} \gamma^{-\left(h_{v}-h_{v^{\prime}}\right) \frac{\eta}{4}}\right] \sum_{\substack{\mathbf{P} \in \mathscr{P}_{\tau} \\
\mid P_{v_{0}}=2 l}}\left[\prod_{v \text { not e.p. }} \gamma^{-\frac{\left|P_{v}\right|}{64}}\right] .
\end{gathered}
$$

Remark: eq. (100) says that a gain $\gamma^{\frac{h}{2}}$ at the scale of the endpoint, see (95), implies a gain $\gamma^{h \frac{1-\eta}{2}}$ at the root scale, as consequence of the fact that the renormalized scaling dimension of all vertices of the trees is strictly positive and $\geq \frac{1}{2}$; this property, which will be extensively used below, is called short memory property.

The sum over $\mathbf{P}$ can be bounded using the following combinatorial inequality: let $\left\{p_{v}, v \in \tau\right\}$, with $\tau \in \mathscr{T}_{h, n}$, be a set of integers such that $p_{v} \leq \sum_{i=1}^{S_{v}} p_{v_{i}}$ for all $v \in \tau$ which are not endpoints; then, if $\alpha>0$,

$$
\prod_{\text {v not e.p. }} \sum_{p_{v}} \gamma^{-\alpha p_{v}} \leq C_{\alpha}^{n} \text {. }
$$


This implies that

$$
\sum_{\substack{\mathbf{P} \in \mathscr{P}_{\tau} \\\left|P_{v_{0}}\right|=2 l}}\left[\prod_{v \text { not e.p. }} \gamma^{-\left|P_{v}\right| \frac{\eta}{32}}\right] \prod_{i=1}^{n} C^{p_{i}}|\lambda|^{\frac{p_{i}}{2}-1} \leq C^{n}|\lambda|^{n} .
$$

Finally

$$
\sum_{\tau \in \mathscr{T}_{h, n} v} \prod_{\text {not e.p. }} \gamma^{-\left(h_{v}-h_{v^{\prime}}\right) \frac{\eta}{4}} \leq C^{n}
$$

as it follows from the fact that the number of non trivial vertices in $\tau$ is smaller than $n-1$ and that the number of trees in $\mathscr{T}_{h, n}$ is bounded by const ${ }^{n}$. Altogether we obtain

$$
\frac{1}{\beta L} \int d \mathbf{x}_{1} \cdots d \mathbf{x}_{l}\left|W_{l}^{(h)}\left(\mathbf{x}_{1}, \ldots, \mathbf{x}_{l}\right)\right| \leq \gamma^{h\left(\frac{3}{2}-\frac{l}{4}\right)} \gamma^{\vartheta h} \sum_{n \geq 1} C^{n}|\lambda|^{n},
$$

where we have set $\vartheta=(1-\eta) / 2$. Moreover we choose $\eta=\frac{1}{2}$ so that $\vartheta=\frac{1}{4}$. Once convergence is established, the limit $L, \beta \rightarrow \infty$ is a straightforward consequence, see for instance section 2 of [8]

In order to complete the proof we need to show the validity of the inductive assumption (86) (87). It is clearly true for $h=1$; moreover, by the bound (101) we get (86). We have finally to prove (87). We can write $g^{(h)}(\mathbf{x})=\left.g^{(h)}\right|_{r=0}(\mathbf{x})+r^{(h)}(\mathbf{x})$ where $\left.g^{(h)}\right|_{r=0}$ is the single scale propagator of the $r=0$ case and $r^{(h)}$ satisfkies

$$
\left|\partial_{0}^{n_{0}} \widetilde{\partial}_{1}^{n_{1}} \widetilde{\boldsymbol{g}}^{(h)}(\mathbf{x})\right| \leq C_{N} \frac{|r| \gamma^{-\frac{h}{2}}}{1+\left[\gamma^{h}\left|x_{0}\right|+\gamma^{\frac{h}{2}}|x|\right]^{N}} \gamma^{h\left(n_{0}+n_{1} / 2\right)}
$$

that is the same bound 61 with an extra $\frac{|r|}{\gamma^{h}}$. We can therefore write

$$
\hat{W}_{2}^{(h)}(0)=\hat{W}_{2, a}^{(h)}(0)+\hat{W}_{2, b}^{(h)}(0)
$$

where $\hat{W}_{2, a}^{(h)}(0)$ is the effective potential of the $r=0$ case. We will show below that $\sum_{h=-\infty}^{1} \hat{W}_{2, a}^{(h)}(0)=0$ and as a consequence $\left|\sum_{h=k}^{1} \hat{W}_{2, a}^{(h)}(0)\right| \leq C|\lambda| \gamma^{(1+\vartheta) k}$ as $\left|\hat{W}_{2, a}^{(h)}(0)\right| \leq C|\lambda| \gamma^{(1+\vartheta) h}$. On the other hand $\left|\hat{W}_{2, b}^{(h)}(0)\right| \leq C|\lambda||r| \gamma^{\vartheta h}$ so that

$$
\gamma^{h-1} \mu_{h-1}=\gamma^{h} \mu_{h}+\hat{W}_{2}^{(h)}(0)
$$

hence $\gamma^{h-1} \mu_{h-1}=\sum_{h=h}^{1} \hat{W}_{2}^{(k)}(0)$ and $\left|\mu_{h}\right| \leq C|\lambda|$. 
It remains to prove that $\sum_{h=-\infty}^{1} \hat{W}_{2, a}^{(h)}(0)=0$. This can be checked noting that in the $r=0$ case it is more natural to consider the following ultraviolet regularization, instead of (16) at $\beta=\infty$

$$
g(\mathbf{x})=\vartheta_{M}\left(x_{0}\right) \int_{-\pi}^{\pi} d k e^{i k x+\varepsilon(k) x_{0}}
$$

with $\varepsilon(k)=\cos (k)-1$ and $\vartheta_{M}\left(x_{0}\right)$ is a smooth function with support in $\left(\gamma^{-M},+\infty\right)$; note that $g(\mathbf{x})$ verifies (17). We can write $g(\mathbf{x})=g^{(u . v .)}(\mathbf{x})+g^{(i . r .)}(\mathbf{x})$ with $g^{(u . v .)}(\mathbf{x})=$ $h\left(x_{0}\right) g(\mathbf{x})$ and $g^{(i . r .)}(\mathbf{x})=\left(1-h\left(x_{0}\right)\right) g(\mathbf{x})$, and $h\left(x_{0}\right)$ a smooth function $=1$ is $\left|x_{0}\right|<1$ and $=0$ if $\left|x_{0}\right|>\gamma$. The integration of the ultraviolet part can be done as in section 3 of [10], writing $\vartheta_{M}\left(x_{0}\right)$ as sum of compact support functions. After that, the limit $M \rightarrow \infty$ can be taken, and we can write $g^{(i . r .)}(\mathbf{x})=\sum_{h=-\infty}^{-1} g^{(h)}(\mathbf{x})$ with

$$
g^{(h)}(\mathbf{x})=\vartheta\left(x_{0}\right)\left(1-h\left(x_{0}\right)\right) \int_{-\pi}^{\pi} d k c_{h}(k) e^{i k x+\varepsilon(k) x_{0}}
$$

with $c_{h}(k)$ a smooth function non vanishing for $\pi \gamma^{h-1} \leq|k| \leq \pi \gamma^{h+1}$; note that $g^{(h)}(\mathbf{x})$ verifies 61 , and the integration of the infrared scales is essentially identical to the one described in this sections. Once all scales are integrated out, we obtain kernels $W_{n, m}^{(-\infty)}$ coinciding with the ones obtained before; however with this choice of the ultraviolet cut-off, $W_{2,0}^{(-\infty)} \equiv 0$ is an immediate consequence of the presence of the $\vartheta_{M}\left(x_{0}\right)$ in the propagator. Indeed the kernels can be written as sum over Feynman graphs, which contain surely a closed fermionic loop or a tadpole (the interaction is local in time).

\subsection{The 2-point Schwinger function in the insulating phase.}

In the case $r=0$ we have $h^{*}=-\infty$ and the integration considered in this section conclude the construction of the effective potential. Similarly, if $r<0$ and $|\lambda|$ is small then $g^{\left(<h^{*}\right)} \equiv 0$, so that again the construction of the effective potential is concluded by the integration on scale $h^{*}$.

In both case the analysis described above can be easily extended to take into account the external fields, that is $\phi \neq 0$ (see for instance section 3.4 of [4] for details in a similar case). The 2-point Schwinger function can be written as, if we define $h_{\mathbf{k}}=\min \{h: \hat{g}(\mathbf{k}) \neq 0\}$

$$
\hat{S}(\mathbf{k})=\sum_{j=h_{\mathbf{k}}}^{h_{\mathbf{k}}+1} Q_{\mathbf{k}}^{(j)} \hat{g}^{(j)}(\mathbf{k}) Q_{\mathbf{k}}^{(j)}-\sum_{j=h_{\mathbf{k}}}^{h_{\mathbf{k}}+1} G^{(j)}(\mathbf{k}) \hat{W}_{2,0}^{(j-1)}(\mathbf{k}) G^{(j)}(\mathbf{k})
$$


where from $(85)\left|\hat{W}_{2,0}^{(j-1)}(\mathbf{k})\right| \leq C|\lambda| \gamma^{(1+\vartheta) j}, Q_{\mathbf{k}}^{(h)}$ is defined inductively by the relation $Q^{(1)}=1$ and

$$
Q_{\mathbf{k}}^{(h)}=1-\hat{W}_{2,0}^{(h)}(\mathbf{k}) g^{(h+1)}(\mathbf{k}) Q_{\mathbf{k}}^{(h+1)}
$$

and

$$
G^{(h+1)}(\mathbf{k})=\sum_{k=h=1}^{1} g^{(k)}(\mathbf{k}) Q_{\mathbf{k}}^{(k)}
$$

so that

$$
\left|Q_{\mathbf{k}}^{(h)}-1\right| \leq C|\lambda| \gamma^{\vartheta h} \quad\left|\hat{G}^{(j)}(\mathbf{k})\right| \leq C|\lambda| \gamma^{-\vartheta h}
$$

Using that

$$
\hat{g}^{(h)}(\mathbf{k})=\frac{f_{h}(\mathbf{k})}{-i k_{0}\left(1+z_{-\infty}\right)+\left(1+\alpha_{-\infty}\right) \frac{k^{2}}{2}}+\hat{r}^{(h)}(\mathbf{k})
$$

where

$$
\left|\hat{r}^{(h)}(\mathbf{k})\right| \leq C \gamma^{(1-\vartheta) h}
$$

so that (14) follows.

\section{Renormalization Group integration: the second regime in the metallic phase.}

We have now to consider the integration of the scales with $h<h^{*}$, that is

$$
e^{-\mathscr{W}(0)}=e^{-\beta L F_{h^{*}}} \int P\left(d \psi^{\left(\leq h^{*}\right)}\right) e^{V^{\left(h^{*}\right)}(\psi)}
$$

where $P\left(d \psi^{\left(\leq h^{*}\right)}\right)$ has propagator given by

$$
g^{\left(\leq h^{*}\right)}(\mathbf{x})=\int d \mathbf{k} \frac{\chi_{\leq h^{*}}(\mathbf{k})}{-i k_{0}\left(1+z_{h^{*}}\right)+\left(1+\alpha_{h^{*}}\right)(\cos k-1)+r+\gamma^{h^{*}} \mu_{h^{*}}}
$$

with $z_{h^{*}}, \alpha_{h^{*}}, v_{h^{*}}=O(\lambda)$.

The denominator of the propagator (113) vanishes in correspondence of the two Fermi momenta and we need a multiscale decomposition. It is convenient to rewrite (112) in the following way

$$
\int P\left(d \psi^{\left(\leq h^{*}\right)}\right) e^{V^{\left(h^{*}\right)}(\psi)}=\int \widetilde{P}\left(d \psi^{\left(\leq h^{*}\right)}\right) e^{V^{\left(h^{*}\right)}(\psi)-\gamma^{h^{*}} v_{h^{*}} \int d \mathbf{x} \psi_{\mathbf{x}}^{+} \psi_{\mathbf{x}}^{-}}
$$

where $\widetilde{P}\left(d \psi^{\left(\leq h^{*}\right)}\right)$ has propagator

$$
g^{\left(\leq h^{*}\right)}(\mathbf{x})=\int d \mathbf{k} e^{i \mathbf{k} \mathbf{x}} \frac{\chi_{\leq h^{*}}(\mathbf{k})}{-i k_{0}\left(1+z_{h^{*}}\right)+\left(1+\alpha_{h^{*}}\right)\left(\cos k-\cos p_{F}\right)}
$$


and

$$
\left(1+\alpha_{h^{*}}\right) \cos p_{F}=\left(1+\alpha_{h^{*}}\right)-r-\gamma^{h^{*}} \mu_{h^{*}}+\gamma^{h^{*}} v_{h^{*}}
$$

Observe that, assuming that also $v_{h^{*}} \leq K|\lambda|$, then we have

$$
C_{-} \sqrt{r} \leq p_{F} \leq C_{+} \sqrt{r}
$$

for $\lambda$ small enough. The strategy of the analysis is the following:

a) we will perform a multiscale analysis of (112). In this analysis we will have to chose $v_{h^{*}}=O(\lambda)$ as function of $p_{F}$ and $\lambda$ to obatin a convergent expansion.

b) at the end of the above construction we will use (116) to obtain the Fermi momentum $p_{F}$ as function of $\lambda$ and $r$.

We can now write

$$
\chi_{\leq h^{*}}(\mathbf{k})=\chi_{\leq h^{*}, 1}(\mathbf{k})+\chi_{\leq h^{*},-1}(\mathbf{k})
$$

where

$$
\chi_{\leq h^{*}, \omega}(\mathbf{k})=\tilde{\vartheta}\left(\omega \frac{k}{p_{F}}\right) \chi_{\leq h^{*}}(\mathbf{k})
$$

where $\omega= \pm 1, \widetilde{\vartheta}$ is a smooth function such that $\widetilde{\vartheta}(k)=1$ for $k>\frac{1}{2}$ and $\widetilde{\vartheta}(k)=0$ for $k<-\frac{1}{2}$ and

$$
\widetilde{\vartheta}(k)+\widetilde{\vartheta}(-k)=1
$$

for every $k$. Thus $\tilde{\vartheta}\left(\frac{k}{p_{F}}\right)$ is equal to 1 in a neighbor of $p_{F}$ and 0 in a neighbor of $-p_{F}$. Clearly $\chi_{\leq h^{*}, \pm 1}(\mathbf{k})$ is a smooth, compact support function and it allows us to write

$$
g^{\left(\leq h^{*}\right)}(\mathbf{x})=\sum_{\omega= \pm 1} e^{i \omega p_{F} x} g_{\omega}^{\left(\leq h^{*}\right)}(\mathbf{x})
$$

where

$$
g_{\omega}^{\left(\leq h^{*}\right)}(\mathbf{x})=\int d \mathbf{k} e^{i\left(\mathbf{k}-\omega \mathbf{p}_{F}\right) \mathbf{x}} \frac{\chi_{\leq h^{*}, \omega}(\mathbf{k})}{-i k_{0}\left(1+z_{h^{*}}\right)+\left(1+\alpha_{h^{*}}\right)\left(\cos k-\cos p_{F}\right)}
$$

with $\mathbf{p}_{F}=\left(0, p_{F}\right)$.

We observe that, if the running coupling constants were not present in the cutoff function $\chi_{\leq h}$, we could have used as a quasi-particle cut-off function

$$
\tilde{\chi}_{\leq h^{*}, \pm 1}(\mathbf{k})=\vartheta( \pm k) \chi_{\leq h^{*}}(\mathbf{k})
$$

where $\vartheta(k)=1$ if $k>0$ and $\vartheta(k)=0$ if $k<0$. Indeed, thanks to (59), this would have made essentially no difference. On the other hand, thanks to (117) and (60), 
we have that $\chi_{\leq h^{*}, \pm 1}$ differs from $\widetilde{\chi}_{\leq h^{*}, \pm 1}$ only for a finite number (not depending on $r$ ) of scales so that this does not modify our qualitative picture. Finally notice that the argument of $\widetilde{\vartheta}$ is not scaled with $\gamma^{-h}$ but only with $p_{F}^{-1}=O\left(\gamma^{-\frac{h^{*}}{2}}\right)$.

The multiscale integration is done exactly as in [4]. The localization operation is defined in the following way

$$
\begin{gathered}
\mathscr{L}_{2} \int d \underline{\mathbf{x}} W_{4}\left(\mathbf{x}_{1}, \mathbf{x}_{2}, \mathbf{x}_{3}, \mathbf{x}_{4}\right) \prod_{i=1}^{4} \psi_{\mathbf{x}_{i}, \omega_{i}}^{\varepsilon_{i}}=\hat{W}_{4}(0) \int d \mathbf{x} \psi_{\mathbf{x}, 1}^{+} \psi_{\mathbf{x}, 1}^{-} \psi_{\mathbf{x},-1}^{+} \psi_{\mathbf{x},-1}^{-} \\
\mathscr{L}_{2} \int d \underline{\mathbf{x}} W_{2}\left(\mathbf{x}_{1}, \mathbf{x}_{2}\right) \psi_{\mathbf{x}_{1}, \omega}^{+} \psi_{\mathbf{x}_{2}, \omega}^{-}=\hat{W}_{2}(0) \int \psi_{\mathbf{x}, \omega}^{+} \psi_{\mathbf{x}, \omega}^{-} d \mathbf{x}+ \\
\partial_{1} \hat{W}_{2}(0) \int \bar{\psi}_{\mathbf{x}, \omega}^{+} \Delta_{1} \psi_{\mathbf{x}, \omega}^{-} d \mathbf{x}+\partial_{0} \hat{W}_{2}(0) \int \psi_{\mathbf{x}, \omega}^{+} \partial_{0} \psi_{\mathbf{x}, \omega}^{-} d \mathbf{x}
\end{gathered}
$$

where

$$
\bar{\Delta}_{1} f(\mathbf{x})=2 \int d \mathbf{k}\left(\cos k-\cos p_{F}\right) e^{i \mathbf{k} \mathbf{x}} \hat{f}(\mathbf{k}) \quad \text { if } \quad f(\mathbf{x})=\int d \mathbf{k} e^{i \mathbf{k x}} \hat{f}(\mathbf{k})
$$

Note that in the kernels $W_{l}$ are included the oscillating factors $e^{i \omega p_{F} x}$ coming form (118).

After the integration of the scale $\psi^{h^{*}}, . . \psi^{h}$ we get

$$
e^{-\mathscr{W}(0)}=e^{-\beta L F_{h}} \int P_{Z_{h}}\left(d \psi^{(\leq h)}\right) e^{V^{h}\left(\sqrt{Z_{h}} \psi\right)}
$$

where $P_{Z_{h}}\left(d \psi^{(\leq h)}\right)$ is the Grasmann integration with propagator $\frac{g_{\omega}^{(\leq h)}}{Z_{h}}$ where

$$
g_{\omega}^{(\leq h)}(\mathbf{x})=\int d \mathbf{k} e^{i\left(\mathbf{k}-\omega \mathbf{p}_{F}\right)} \frac{\chi_{\leq h, \omega}(\mathbf{k})}{\left(1+z_{h^{*}}\right) i k_{0}+\left(1+\alpha_{h^{*}}\right)\left(\cos k-\cos p_{F}\right)}
$$

We can now write

$$
\int P_{Z_{h}}\left(d \psi^{(\leq h)}\right) e^{V^{h}\left(\sqrt{Z_{h}} \psi\right)}=\int \widetilde{P}_{Z_{h-1}}\left(d \psi^{(\leq h)}\right) e^{\widetilde{V}^{h}\left(\sqrt{Z_{h}} \psi\right)}
$$

where

$$
\begin{aligned}
\mathscr{L}_{2} \widetilde{V}^{h}= & l_{h} \int d \mathbf{x} \psi_{\mathbf{x}, 1}^{+} \psi_{1, \mathbf{x}}^{-} \psi_{\mathbf{x},-1}^{+} \psi_{-1, \mathbf{x}}^{-}+\left(a_{h}-z_{h}\right) \sum_{\omega} \int d \mathbf{x} \psi_{\omega, \mathbf{x}}^{+} \partial \psi_{\omega, \mathbf{x}}+ \\
& n_{h} \int d \mathbf{x} \psi_{\omega, \mathbf{x}}^{+} \psi_{\omega, \mathbf{x}}
\end{aligned}
$$

while $\widetilde{P}_{h}\left(d \psi^{(\leq h)}\right)$ is the integration with propagator identical to (122) but with $\chi_{\leq h, \omega}(\mathbf{k})$ replaced by $\frac{\chi_{\leq h, \omega}(\mathbf{k})}{\widetilde{Z}_{h-1}(\mathbf{k})}$ with

$$
\widetilde{Z}_{h-1}(\mathbf{k})=Z_{h}+\chi_{\leq h, \omega}(\mathbf{k}) Z_{h} z_{h}
$$


Setting $Z_{h-1}=\widetilde{Z}_{h-1}(0)$, we can finally write

$$
\int P_{Z_{h}}\left(d \psi^{(\leq h)}\right) e^{V^{h}\left(\sqrt{Z_{h}} \psi\right)}=e^{-\beta L e_{h}} \int P_{Z_{h-1}}\left(d \psi^{(\leq h-1)}\right) \int \widetilde{P}_{Z_{h-1}}\left(d \psi^{(h)}\right) e^{-\bar{V}^{(h)}\left(\psi^{(\leq h)}\right)}
$$

where $\widetilde{P}_{Z_{h-1}}$ is the integration with propagator $\frac{\widetilde{g}_{\omega}^{(h)}}{Z_{h-1}}$

$$
\widetilde{g}_{\omega}^{(h)}(\mathbf{x})=\int d \mathbf{k} e^{i \mathbf{x}\left(\mathbf{k}-\omega \mathbf{p}_{F}\right)} \frac{\widetilde{f}_{h, \omega}(\mathbf{k})}{\left(1+z_{h^{*}}\right) i k_{0}+\left(1+\alpha_{h^{*}}\right)\left(\cos k-\cos p_{F}\right)}
$$

where

$$
\widetilde{f}_{h, \omega}(\mathbf{k})=Z_{h-1}\left[\frac{\chi_{\leq h, \omega}(\mathbf{k})}{\widetilde{Z}_{h-1}(\mathbf{k})}-\frac{\chi_{\leq h-1, \omega}(\mathbf{k})}{Z_{h-1}}\right] .
$$

Finally we have

$$
\bar{V}^{(h)}\left(\psi^{(\leq h)}\right)=\widetilde{V}^{(h)}\left(\sqrt{\frac{Z_{h}}{Z_{h-1}}} \psi^{(\leq h)}\right)
$$

so that

$$
\begin{array}{r}
\mathscr{L}_{2} \widetilde{V}^{h}=\lambda_{h} \int d \mathbf{x} \psi_{\mathbf{x}, 1}^{+} \psi_{1, \mathbf{x}}^{-} \psi_{\mathbf{x},-1}^{+} \psi_{-1, \mathbf{x}}^{-}+\delta_{h} \sum_{\omega} \int d \mathbf{x} \psi_{\omega, \mathbf{x}}^{+} \partial \psi_{\omega, \mathbf{x}}+ \\
\gamma^{h} v_{h} \sum_{\omega} \int d \mathbf{x} \psi_{\omega, \mathbf{x}}^{+} \psi_{\omega, \mathbf{x}}
\end{array}
$$

with

$$
\gamma^{h} v_{h}=\frac{Z_{h}}{Z_{h-1}} n_{h} \quad \delta_{h}=\frac{Z_{h}}{Z_{h-1}}\left(a_{h}-z_{h}\right) \quad \lambda_{h}=\left(\frac{Z_{h}}{Z_{h-1}}\right)^{2} l_{h}
$$

We can now prove the following:

Lemma 3.1. For $h \leq h^{*}$, every $N$ and $\lambda$ small enough we have

$$
\left|\partial_{0}^{n_{0}} \partial_{1}^{n_{1}} \widetilde{g}_{\omega}^{(h)}(\mathbf{x})\right| \leq C_{N} \frac{v_{F}^{-1} \gamma^{h}}{1+\left[\gamma^{h}\left|x_{0}\right|+v_{F}^{-1} \gamma^{h}|x|\right]^{N}} \gamma^{h\left(n_{0}+n_{1}\right)} v_{F}^{-n_{1}}
$$

with $v_{F}=\sin \left(p_{F}\right)=O\left(r^{\frac{1}{2}}\right)$.

Proof. We can write

$$
\cos k-\cos p_{F}=\cos p_{F}\left(\cos \left(k-\omega p_{F}\right)-1\right)+\omega v_{F} \sin \left(k-\omega p_{F}\right)
$$

Using (62) it easily follows that

$$
\left|\sin \left(k-p_{F}\right)\right| \leq C \gamma^{h} r^{-\frac{1}{2}}
$$


and thus $\left|k-p_{F}\right| \leq C \gamma^{h} r^{-\frac{1}{2}}$. For this case we get

$$
\int \widetilde{\vartheta}(k) \chi_{\leq h}(\mathbf{k}) d \mathbf{k} \leq C r^{-\frac{1}{2}} \gamma^{2 h}
$$

The analogous of (65) for the present Lemma is

$$
\left|x_{0}^{N_{0}} x^{N_{1}} \widetilde{g}_{\omega}^{(h)}(\mathbf{x})\right| \leq C \gamma^{h\left(1-N_{0}-N_{1}\right)} r^{\frac{N_{1}-1}{2}}
$$

To prove it we observe that (67) and (68) remain true. Indeed the only difference arise due to the presence of $\widetilde{\vartheta}$. But this does not change the estimates since its derivative gives a smaller factor $O\left(\gamma^{-\frac{h^{*}}{2}}\right)$ as compared to the factor $O\left(\sqrt{r} \gamma^{-h}\right)$ coming from the derivative of $\widetilde{f}_{h, \omega}$. Again we use (69) for $P_{1} \leq N_{1} / 2$ together with

$$
\gamma^{-h\left(1+N_{0}+P_{1}\right)} \leq \gamma^{-h\left(1+N_{0}+N_{1}\right)} r^{N_{1}-P_{1}} \leq \gamma^{-h\left(1+N_{0}+N_{1}\right)} r^{\frac{N_{1}}{2}}
$$

Reasoning as before, for $P_{1}>N_{1} / 2$ we get

$$
\prod_{i=i}^{P_{1}} \frac{d^{p_{1}}}{d k^{p_{i}}} \sin (k) \leq C \gamma^{\left(2 P_{1}-N_{1}\right) h} r^{\frac{N_{1}}{2}-P_{1}}
$$

Observing that

$$
\gamma^{-h\left(1+N_{0}+P_{1}\right)} \gamma^{\left(2 P_{1}-N_{1}\right) h} r^{\frac{N_{1}}{2}-P_{1}}=\gamma^{-h\left(1+N_{0}+N_{1}\right)} r^{\frac{N_{1}}{2}}\left(\frac{\gamma^{h}}{r}\right)^{P_{1}} \leq C \gamma^{-h\left(1+N_{0}+N_{1}\right)} r^{\frac{N_{1}}{2}}
$$

and collecting we get

$$
\left|\partial_{0}^{N_{0}} \partial_{1}^{N_{1}} \hat{g}^{(h)}(\mathbf{k})\right| \leq \gamma^{-\left(1+N_{0}+N_{1}\right) h} r^{\frac{N_{1}}{2}}
$$

The Lemma follows easily combining the above estimate with (127) and the analogous of (72).

Again the effective potential can be written as a sum over trees similar to the previous ones but with the following modifications:

1. We associate a label $h \leq h^{*}$ with the root.

2. With each endpoint $v$ we associate one of the monomials contributing to $\mathscr{R}_{2} \mathscr{V}^{\left(h^{*}\right)}\left(\psi^{\left(\leq h_{v}-1\right)}\right)$ or one of the terms contributing to $\mathscr{L}_{2} \mathscr{V}^{\left(h_{v}\right)}\left(\psi^{\left(\leq h_{v}-1\right)}\right)$.

The main result of this section is the following Lemma. 
Lemma 3.2. Assume that

$$
\left|\lambda_{k}\right|,\left|\delta_{k}\right| \leq C v_{F}|\lambda| \quad\left|v_{k}\right| \leq C|\lambda|
$$

than there exists a constants $\lambda_{0}>0$, independent of $\beta, L$ and $r$, such that, for $h<h^{*}$, the kernels $W_{l}^{(h)}$ are analytic functions of $\lambda$ for $|\lambda| \leq \lambda_{0}$. Moreover they satisfy

$$
\frac{1}{\beta L} \int d \mathbf{x}_{1} \cdots d \mathbf{x}_{l}\left|W_{l}^{(h)}\left(\mathbf{x}_{1}, \ldots, \mathbf{x}_{l}\right)\right| \leq \gamma^{h\left(2-\frac{l}{2}\right)} v_{F}^{\frac{l}{2}-1}(C|\lambda|)^{\max (1, l-1)} .
$$

Proof. The proof of this Lemma follows closely the line of [4]. The only major difference is the presence of the small factors in (126). We will report only the modification of the proof needed to deal with those factors.

We start noting that the analogous of the bound (88) becomes

$$
\begin{aligned}
& \frac{1}{\beta L} \int d \mathbf{x}_{1} \cdots d \mathbf{x}_{l}\left|W_{l}^{(h)}\left(\mathbf{x}_{1}, \ldots, \mathbf{x}_{l}\right)\right| \leq \\
& \sum_{n \geq 1} \sum_{\tau \in \mathscr{T}, n} \sum_{\substack{\mathbf{P} \in \mathscr{P} \\
\left|P_{v_{0}}\right|=l}} \sum_{T \in \mathbf{T}} C^{n}\left[\prod_{v \text { not e.p. }} \frac{1}{s_{v} !} \gamma^{h_{v}\left(\sum_{i=1}^{s_{v}} \frac{\left|P_{v_{i}}\right|}{2}-\frac{\left|P_{v}\right|}{2}-2\left(s_{v}-1\right)\right)}\right] \\
& {\left[\prod_{v \text { not e.p. }}\left(\frac{1}{v_{F}}\right)^{\sum_{i=1}^{s_{v}} \frac{\left|P_{v_{i}}\right|}{2}-\frac{\left|P_{v}\right|}{2}-\left(s_{v}-1\right)}\right]\left[\prod_{v \text { not e.p. }} \gamma^{-\left(h_{v}-h_{v^{\prime}}\right) z_{2}\left(P_{v}\right)}\right]} \\
& {\left[\prod_{v \text { e.p.; } ; \in I^{R},\left|I_{v}\right| \geq 6}|\lambda| \gamma^{h^{*}\left(\frac{3}{2}-\frac{\left|I_{v}\right|}{4}\right)}\right]\left[\prod_{v \text { e.p.; } ; \in I^{R},\left|I_{v}\right|=2,4}|\lambda| \gamma^{h^{*}\left(\frac{3}{2}-\frac{\left|I_{v}\right|}{4}\right)+z_{2}\left(P_{v}\right)\left(h_{v^{\prime}}-h^{*}\right)}\right]} \\
& {\left[\prod_{v \text { e.p. } ; v \in I^{\lambda}}|\lambda| v_{F}\right]\left[\prod_{v \text { e.p.; } ; \in I^{v, \delta}}|\lambda| \gamma^{h_{v^{\prime}}}\right]\left[\prod_{i=1}^{n} C^{p_{i}}\right]}
\end{aligned}
$$

where:

1. the last factor keeps into account the presence of the factors $Z_{h} / Z_{h-1}$;

2. the factor $\left(\frac{1}{v_{F}}\right)^{\frac{\left|P_{v_{i}}\right|}{2}-\frac{\left|P_{V}\right|}{2}-\left(s_{v}-1\right)}$ comes from the bound on the Gram determinant and the fact that $\left|g^{h}(\mathbf{x})\right| \leq \frac{\gamma^{h}}{v_{F}}$;

3. $z_{2}\left(P_{v}\right)=1$ for $\left|P_{v}\right|=4$ and $z_{2}\left(p_{v}\right)=2$ for $\left|P_{v}\right|=2$;

4. $I^{R}$ is the set of endpoints associated to $\mathscr{R} \mathscr{V}^{\left(h^{*}\right)}$ and the factor $\gamma^{h^{*}\left(\frac{3}{2}-\frac{\left|I_{V}\right|}{4}\right)}$ comes from the bound (85); 
5. $I^{\lambda}$ is the set of end-points associated to $\lambda_{k}$ and the factor $v_{F}$ comes from (131);

6. $I^{\delta}$ is the set of end-points associated to $\delta_{k}$ and the derivative in (125) produces an extra $\gamma^{h_{v^{\prime}}} / v_{F}$;

7. $I^{v}$ is the set of end-points associated to $v_{k}$ and the factor $\gamma^{h_{v^{\prime}}}$ comes from (125).

Proceeding like in the proof of Lemma 2.2 using (91) we get

$$
\begin{aligned}
& \frac{1}{\beta L} \int d \mathbf{x}_{1} \cdots d \mathbf{x}_{l}\left|W_{l}^{(h)}\left(\mathbf{x}_{1}, \ldots, \mathbf{x}_{l}\right)\right| \leq \\
& \sum_{n \geq 1} \sum_{\tau \in \mathscr{T}, n} \sum_{\substack{\mathbf{P} \in \mathscr{P}_{\tau} \\
\left|P_{v_{0}}\right|=l}} \sum_{T \in \mathbf{T}} C^{n} \gamma^{h\left(2-\frac{1}{2}\left|P_{v_{0}}\right|+\frac{1}{2}\left|I_{v_{0}}\right|-2 n\right)} \\
& {\left[\prod_{v \text { not e.p. }} \frac{1}{s_{v} !} \gamma^{\left(h_{v}-h_{v^{\prime}}\right)\left(2-\frac{\left|P_{v}\right|}{2}+\frac{||_{v} \mid}{2}-2 n(v)+z_{2}\left(P_{v}\right)\right)}\right]} \\
& {\left[\prod_{v \text { e.p.; } ; v \in I^{R}}|\lambda| \gamma^{h_{v^{\prime}}\left(\frac{3}{2}-\frac{\left|I_{v}\right|}{4}\right)}\right]\left[\prod_{v \text { e.p.; } ; \in I^{\lambda}}|\lambda| v_{F}\right]\left[\prod_{v \text { e.p.; } ; \in I^{v, \delta}}|\lambda| \gamma^{h_{v^{\prime}}}\right]\left[\prod_{i=1}^{n} C^{p_{i}}\right]} \\
& {\left[\prod_{v \text { not e.p. }}\left(\frac{1}{v_{F}}\right)^{\left(\sum_{i=1}^{s_{v}} \frac{\left|P_{v_{i}}\right|}{2}-\frac{\left|P_{v}\right|}{2}-\left(s_{v}-1\right)\right)}\right]}
\end{aligned}
$$

Finally using (93) we arrive to

$$
\begin{aligned}
\frac{1}{\beta L} \int d \mathbf{x}_{1} \cdots d \mathbf{x}_{l}\left|W_{l}^{(h)}\left(\mathbf{x}_{1}, \ldots, \mathbf{x}_{l}\right)\right| \leq & \sum_{n \geq 1} \sum_{\tau \in \mathscr{T}, n} \sum_{\substack{\mathbf{P} \in \mathscr{P}_{\tau} \\
\left|P_{v_{0}}\right|=l}} \sum_{T \in \mathbf{T}} C^{n} \gamma^{h\left(2-\frac{1}{2}\left|P_{v_{0}}\right|\right)}\left[\prod_{v \text { not e.p. }} \frac{1}{s_{v} !} \gamma^{\left(h_{v}-h_{v^{\prime}}\right)\left(2-\frac{\left|P_{v}\right|}{2}+z_{2}\left(P_{v}\right)\right)}\right] \\
& {\left[\prod_{v \text { e.p. }, v \in I^{R}} \gamma^{h_{v^{\prime}}\left(-\frac{1}{2}+\frac{\left|I_{v}\right|}{4}\right)}\right]\left[\prod_{v}|\lambda| v_{F}\right]\left[\prod_{v \text { e.p.; } ; \in I^{\lambda}}|\lambda|\right]\left[\prod_{i=1}^{n} C^{p_{i}}\right] } \\
& {\left[\prod_{v \text { not e.p.; } ; \in I^{v, \delta}}\left(\frac{1}{v_{F}}\right)^{\left(\sum_{i=1}^{s_{v}} \frac{\left|P_{v^{\prime}}\right|}{2}-\frac{\left|P_{v}\right|}{2}-\left(s_{v}-1\right)\right)}\right] }
\end{aligned}
$$

Because $\gamma^{h_{v}^{\prime}} \leq \gamma^{h^{*}} \leq v_{F}^{2}$ and $\left|I_{v}\right| \geq 2$ we have

$$
\gamma^{h_{\nu^{\prime}}\left(-\frac{1}{2}+\frac{\left|I_{v}\right|}{4}\right)} \leq v_{F}^{-1+\frac{\left|V_{v}\right|}{2}}
$$


so that

$$
\begin{aligned}
& \frac{1}{\beta L} \int d \mathbf{x}_{1} \cdots d \mathbf{x}_{l}\left|W_{l}^{(h)}\left(\mathbf{x}_{1}, \ldots, \mathbf{x}_{l}\right)\right| \leq \\
& \sum_{n \geq 1} \sum_{\tau \in \mathscr{T}, n} \sum_{\substack{\mathbf{P} \in \mathscr{P}_{\tau} \\
\left|P_{v_{0}}\right|=l}} \sum_{T \in \mathbf{T}} C^{n} \gamma^{h\left(2-\frac{1}{2}\left|P_{v_{0}}\right|\right)}\left[\prod_{v \text { not e.p. }} \frac{1}{s_{v} !} \gamma^{\left(h_{v}-h_{v^{\prime}}\right)\left(2-\frac{\left|P_{v}\right|}{2}+z_{2}\left(P_{v}\right)\right)}\right] \\
& {\left[\prod_{v \text { e.p. }, v \in I^{R}, I^{\lambda}} v_{F}^{-1+\frac{\left|I_{v}\right|}{2}}\right]\left[\prod_{v \text { not e.p. }}\left(\frac{1}{v_{F}}\right)^{\left(\sum_{i=1}^{s_{v}} \frac{\left|P_{v}\right|}{2}-\frac{\left|P_{v}\right|}{2}-\left(s_{v}-1\right)\right)}\right]|\lambda|^{n}\left[\prod_{i=1}^{n} C^{p_{i}}\right]}
\end{aligned}
$$

For $v \in I^{\delta}, I^{v}$, one has $\left|I_{v}\right|=2$ so that $v_{F}^{-1+\frac{\left|I_{v}\right|}{2}}=1$, and we can write

$$
\left[\prod_{v \text { e.p. }, v \in I^{R}, I^{\lambda}} v_{F}^{-1+\frac{\left|V_{v}\right|}{2}}\right]=\left[\prod_{v \text { e.p. }} v_{F}^{-1+\frac{\left|I_{v}\right|}{2}}\right]=v_{F}^{-n+\sum_{v \text { e.p }} \frac{\left|I_{v}\right|}{2}}
$$

Using that

$$
\sum_{v}\left(s_{v}-1\right)=n-1 \quad \sum_{v \text { e.p. }}\left|I_{v}\right|=l+\sum_{v} \sum_{i=1}^{S_{v}}\left(\left|P_{v_{i}}\right|-\left|P_{v}\right|\right)
$$

we get

$$
\begin{aligned}
& \prod_{v \text { e.p. }} v_{F}^{-1} \prod_{v \text { not e.p. }}\left(\frac{1}{v_{F}}\right)^{-\left(s_{v}-1\right)}=v_{F}^{-1} \\
& \prod_{v \text { e.p. }} v_{F}^{\frac{\left|V_{v}\right|}{2}} \prod_{v \text { not e.p. }}\left(\frac{1}{v_{F}}\right)^{\left(\sum_{i=1}^{s_{v}} \frac{\left|P_{v_{i}}\right|}{2}-\frac{\left|P_{v}\right|}{2}\right)}=v_{F}^{\frac{l}{2}}
\end{aligned}
$$

Collecting these estimates we get

$$
\begin{aligned}
& \frac{1}{\beta L} \int d \mathbf{x}_{1} \cdots d \mathbf{x}_{l}\left|W_{l}^{(h)}\left(\mathbf{x}_{1}, \ldots, \mathbf{x}_{l}\right)\right| \leq \\
& v_{F}^{\frac{l}{2}-1} \sum_{n \geq 1} \sum_{\tau \in \mathscr{T}, n} \sum_{\substack{\mathbf{P} \in \mathscr{P}_{\tau} \\
\left|P_{v_{0}}\right|=l}} \sum_{T \in \mathbf{T}} C^{n} \gamma^{h\left(2-\frac{1}{2}\left|P_{v_{0}}\right|\right)}\left[\prod_{v \text { not e.p. }} \frac{1}{s_{v} !} \gamma^{\left(h_{v}-h_{v^{\prime}}\right)\left(2-\frac{\left|P_{v}\right|}{2}+z_{2}\left(P_{v}\right)\right)}\right] \\
& |\lambda|^{n}\left[\prod_{i=1}^{n} C^{p_{i}}\right]
\end{aligned}
$$

Performing the sums as in the previous section we prove (132). 


\section{Remarks.}

- Observe that, for $h \geq h^{*}$, bound (85) says that the $L_{1}$ norm of the effective potential is $O\left(\gamma^{h(3 / 2-l / 4)}\right)$ while, for $h \leq h^{*}$, bound (132) says that the $L_{1}$ norm of the effective potential is $O\left(\gamma^{h(2-l / 2)} v_{F}^{\frac{l}{2}-1}\right)$; the two bounds coincide of course at $h \simeq h^{*}$ since $\gamma^{h^{*}} \sim r, v_{F} \sim \sqrt{r}$ so that $r^{(2-l / 2)} r^{\frac{l}{4}-\frac{1}{2}}=r^{\frac{3}{2}-\frac{l}{4}}$.

- The fact that the Fermi velocity vanishes as $r$ approaches 0 produces the "dangerous" factor $\left(\frac{1}{v_{F}}\right)^{\frac{\left|P_{V_{i}}\right|}{2}-\frac{\left|P_{V}\right|}{2}-\left(s_{v}-1\right)}$ in (134) which is diverging as $r \rightarrow 0$. This is compensated by the extra factors of $v_{F}$ associated to the difference between the scaling dimensions the first and second regime, that is

$$
\left[\prod_{v \text { e.p. }} \gamma^{h_{v^{\prime}}\left(\frac{3}{2}-\frac{\left|V_{v}\right|}{4}\right)}\right]=\left[\prod_{v \text { e.p. }} \gamma^{h_{\nu^{\prime}}\left(2-\frac{\left|V_{v}\right|}{2}\right)}\right]\left[\prod_{v \text { e.p. }} \gamma^{h_{v^{\prime}}\left(-\frac{1}{2}+\frac{\left|V_{v}\right|}{4}\right)}\right]
$$

\subsection{The flow of the running coupling constants}

We now prove by induction that, for $h \leq h^{*}$ and $\vartheta=\frac{1}{4}$ we have

$$
\left|\lambda_{h}\right| \leq C|\lambda| r^{\frac{1}{2}+\vartheta}, \quad\left|\delta_{h}\right| \leq C|\lambda| r^{\frac{1}{2}+\vartheta} \quad\left|v_{h}\right| \leq C|\lambda| \gamma^{\vartheta h}
$$

First we check that (131) is true for $h=h^{*}$. By definition of the $\mathscr{L}_{2}$ operation

$$
\lambda_{h^{*}}=\lambda\left[\hat{v}(0)-\hat{v}\left(2 p_{F}\right)\right]+O\left(\lambda^{2} \gamma^{h^{*}\left(\frac{1}{2}+\vartheta\right)}\right)
$$

where the second term in the r.h.s comes from (85); as $\hat{v}(k)$ is even the first term is $O(r)$ so that surely $\lambda_{h^{*}}$ vanishes as $O\left(r^{\frac{1}{2}+\vartheta}\right)$. Moreover from (85), taking into account that a derivative $\partial_{1}$ gives an extra $\gamma^{-h / 2}$, that is

$$
\int d \mathbf{x}\left|\partial_{1} W_{2}^{\left(h^{*}\right)}(\mathbf{x})\right| \leq C|\lambda| \gamma^{h^{*}\left(\frac{1}{2}+\vartheta\right)}
$$

we get

$$
\left|\delta_{h^{*}}\right| \leq C \gamma^{h\left(\frac{1}{2}+\vartheta\right)}|\lambda| \leq C|\lambda| r^{\frac{1}{2}+\vartheta}
$$

The flow of $v_{h}$ is given by

$$
v_{h-1}=\gamma v_{h}+\beta_{v}^{(h)}\left(\vec{v}_{h}, \ldots, \vec{v}_{0}\right)
$$

where $\vec{v}_{h}=\left(\lambda_{h}, \delta_{h}, v_{h}\right)$. We can decompose the propagator as

$$
\widetilde{g}_{\omega}^{(h)}(\mathbf{x})=g_{\omega, L}^{(h)}(\mathbf{x})+r_{\omega}^{(h)}(\mathbf{x})
$$


where

$$
g_{\omega, L}^{(h)}(\mathbf{x})=\int d \mathbf{k} e^{i \mathbf{k} \mathbf{x}} \frac{\widetilde{f}_{h}(\mathbf{k})}{-i k_{0}+\omega v_{F} k}
$$

and $\widetilde{f}_{h}$ has support contained in $C \gamma^{h-1} \leq \sqrt{k_{0}^{2}+v_{F}^{2} k^{2}} \leq C \gamma^{h+1}$. Moreover, for every $N$, we have

$$
\left|r_{\omega}^{(h)}(\mathbf{x})\right| \leq\left(\frac{\gamma^{h}}{v_{F}}\right)^{3} \frac{C_{N}}{1+\gamma^{h}\left(\left|x_{0}\right|+v_{F}^{-1}|x|\right)^{N}}
$$

that is the bound for $r_{\omega}^{(h)}(\mathbf{x})$ has an extra factor $\gamma^{2 h} / v_{F}^{2} \leq \gamma^{h}$ with respect to the bound (126) for $\widetilde{g}_{\omega}^{(h)}(\mathbf{x})$.

In the expansion for $\beta_{v}^{(h)}$ studied in the previous subsection, we can decompose every propagator as in (146) and collect all the term that contains only $g_{L, \omega}^{(h)}$ and that come from trees with no end-points associated to $\mathscr{R} \mathscr{V}^{\left(h^{*}\right)}$; this sum vanish due to parity. Therefore $\beta_{v}^{(h)}=O\left(\lambda \gamma^{\vartheta h}\right)$ and by iteration

$$
v_{h-1}=\gamma^{-h+h^{*}}\left[v_{h^{*}}+\sum_{k=h}^{h^{*}} \gamma^{k-h^{*}} \beta_{v}^{(k)}\right] .
$$

Thus we can choose $v_{h^{*}}$ so that

$$
v_{h^{*}}=-\sum_{k=-\infty}^{h^{*}} \gamma^{k-h^{*}} \beta_{v}^{(k)}
$$

This implies that

$$
v_{h-1}=\gamma^{-h+h^{*}}\left[-\sum_{k=-\infty}^{h} \gamma^{k-h^{*}} \beta_{v}^{(k)}\right]
$$

and $\left|v_{h}\right| \leq C|\lambda| \gamma^{\vartheta h}$.

We now study the flow equations for $\lambda_{h}$ and $\delta_{h}$ with $h<h^{*}$

$$
\begin{aligned}
& \lambda_{h-1}=\lambda_{h}+\beta_{\lambda}^{(h)}\left(\vec{v}_{h}, \ldots, \vec{v}_{0}\right) \\
& \delta_{h-1}=\delta_{h}+\beta_{\delta}^{(h)}\left(\vec{v}_{h}, \ldots, \vec{v}_{0}\right)
\end{aligned}
$$

where we have redefined $\delta_{0}$ as to include the sum $\widetilde{\delta}_{0}$ of the terms $O(\lambda)$, which satisfies

$$
\left|\widetilde{\delta}_{0}\right| \leq C\left|\int d \mathbf{k} k \partial^{2} v\left(\mathbf{k}+\left(\omega-\omega^{\prime}\right) \mathbf{p}_{F}\right) g_{\omega}^{\leq h^{*}}(\mathbf{k})\right|
$$

where one derivative over $v$ comes from the $\mathscr{R}_{1}$ operation and the other from the definition of $\delta$. Observe that

$$
\left|\widetilde{\delta}_{0}\right| \leq C \sum_{k \leq h^{*}} v_{F}^{-2} \gamma^{2 h} \leq C|\lambda| r
$$


since $v_{F} k \leq C \gamma^{h}$ in the support of $f_{h}$.

Again we can use (146) and decompose the beta function for $\alpha=\lambda, \delta$ as

$$
\beta_{\alpha}^{(h)}\left(\vec{v}_{h}, \ldots, \vec{v}_{0}\right)=\bar{\beta}_{\alpha}^{(h)}\left(\lambda_{h}, \delta_{h}, \ldots, \lambda_{0}, \delta_{0}\right)+\beta_{\alpha, R}^{(h)}\left(\vec{v}_{h}, \ldots, \vec{v}_{0}\right)
$$

where $\bar{\beta}_{\alpha}^{(h)}$ contains only propagators $g_{\omega, L}^{(h)}(\mathbf{x})$ and end-points to which is associated $\lambda_{k}, \delta_{k}$. Therefore $\beta_{\alpha, R}^{(h)}$ contains either a propagator $r_{\omega, L}^{(h)}(\mathbf{x})$, a $v_{k}$ or an irrelevant term. Observe that

1. Terms containing a propagator $r_{h}$ or a factor $v_{h}$ have an extra $\gamma^{\vartheta h}$ in their bounds, therefore by an argument similar to the one used in (70) (short memory property) they can be bounded as $O\left(v_{F} \gamma^{\vartheta h}\right)$. The factor $v_{F}$ comes from the factor $v_{F}^{l / 2-1}$ in (132) when $\alpha=\lambda$, and from the derivative $\partial_{1}$ in the case $\alpha=\delta$.

2. The terms containing an irrelevant end-points associated to a term $\mathscr{R} V^{\left(h^{*}\right)}$ have an extra $\gamma^{\vartheta h^{*}}$ (coming from (59)) and an extra $\gamma^{\vartheta\left(h-h^{*}\right)}$ for the short memory property; therefore they can be bound as $O\left(v_{F} \gamma^{\vartheta h}\right)$. The origin of the factor $v_{F}$ is the same as in the previous point.

In conclusion

$$
\left|\beta_{\alpha, R}^{(h)}\right| \leq C v_{F} \lambda^{2} \gamma^{\vartheta h}
$$

From (147) it is easy to see that

$$
\begin{aligned}
& \bar{\beta}_{\lambda}^{(h)}\left(\lambda_{h}, \delta_{h}, \ldots, \lambda_{0}, \delta_{0}\right)=v_{F} \hat{\beta}_{\lambda}^{(h)}\left(\frac{\lambda_{h}}{v_{F}}, \frac{\delta_{h}}{v_{F}}, \ldots, \frac{\lambda_{0}}{v_{F}}, \frac{\delta_{0}}{v_{F}}\right) \\
& \bar{\beta}_{\delta}^{(h)}\left(\lambda_{h}, \delta_{h}, \ldots, \lambda_{0}, \delta_{0}\right)=v_{F} \hat{\beta}_{\delta}^{(h)}\left(\frac{\lambda_{h}}{v_{F}}, \frac{\delta_{h}}{v_{F}}, . ., \frac{\lambda_{0}}{v_{F}}, \frac{\delta_{0}}{v_{F}}\right)
\end{aligned}
$$

where $\hat{\beta}_{\lambda}^{(h)}\left(\lambda_{d}, \delta_{h}, . ., \lambda, \delta_{0}\right)$ is the beta function of a Luttinger model with $v_{F}=1$. It has been proved in [5] that

$$
\left|\hat{\beta}_{\lambda}^{(h)}\left(\lambda_{d}, \delta_{h}, . ., \lambda_{0}, \delta_{0}\right)\right| \leq C\left[\max \left(\left|\lambda_{k}\right|,\left|\delta_{k}\right|\right)\right]^{2} \gamma^{\vartheta\left(h-h^{*}\right)}
$$

therefore assuming by induction that $\left|\lambda_{k}\right|,\left|\delta_{k}\right| \leq 2|\lambda| r^{\frac{1}{2}+\vartheta}$ for $k \geq h$ we get

$$
\left|\bar{\beta}_{\alpha}^{(h)}\left(\lambda_{h}, \delta_{h}, \ldots, \lambda_{0}, \delta_{0}\right)\right| \leq 4 C v_{F} \lambda^{2} r^{1+2 \vartheta} \gamma^{\vartheta h} v_{F}^{-2} r^{-\vartheta} \leq 4 C v_{F} \lambda^{2} \gamma^{\vartheta h} r^{\vartheta}
$$

Thus

$$
\left|\lambda_{h-1}\right| \leq\left|\lambda_{h^{*}}\right|+\sum_{k=h}^{h^{*}} 4 C v_{F} \lambda^{2} \gamma^{\vartheta h} r^{\vartheta} \leq 2|\lambda| r^{\frac{1}{2}+2 \vartheta}
$$


and the same is true for $\delta_{h}$.

Moreover we have

$$
\frac{Z_{h-1}}{Z_{h}}=1+\beta_{z}^{(h)}
$$

so that

$$
\gamma^{\eta}=1+\beta^{-\infty}\left(\frac{\lambda_{-\infty}}{v_{F}}\right)
$$

where $\beta^{-\infty}$ is the beta function with $v_{F}=1$; therefore

$$
Z_{h}=\gamma^{\eta\left(h-h^{*}\right)}(1+A(\lambda))
$$

with $|A(\lambda)| \leq C|\lambda|$. Observe that $\eta=O\left(\lambda^{2} r^{4 \vartheta}\right)$, hence is vanishing as $r \rightarrow 0$ as $O\left(\lambda^{2} r\right)$.

Finally the inversion problem for $p_{F}$ can be studied as in section 2.9 of [8]. The analysis for the Schwinger function is done in a way similar to the one in section 3 above.

\section{References}

[1] S. Sachdev. Quantum Phase Transitions,Cambridge University Press (2011)

[2] M.Garst, D.S. Novikov, A . Stern, L.I.Glazman Phys. Rev. B 77, 035128 (2008)

[3] D. Mattis, E. Lieb J. Math. Phys. 4,5 1154 (1963)

[4] G. Benfatto, V. Mastropietro Rev. Math. Phys. 13, 1323-1435, (2001).

[5] G. Benfatto,V. Mastropietro, Comm. Math. Phys. 231, 97-134, (2002).

[6] G. Benfatto, V. Mastropietro, Comm. Math. Phys. 258, 609-655, (2005).

[7] D.M.Haldane Phys.Rev.Lett. 45, 1358-1362, (1980).

[8] G.Benfatto, P.Falco, V.Mastropietro Comm. Math.Phys 2014

[9] G. Gallavotti Rev. Mod. Phys. 57 (1985) 471

[10] Benfatto G., Gallavotti G, Procacci, A, Scoppola B: Comm. Math. Phys. 160, 93 (1994).

[11] D. Brydges. Les Houches ed. Noth Holland (1984) 
[12] K. Gawedzki, A. Kupiainen Comm.Math. Phys. 102, 1 (1985)

[13] A. Lesniewski, Comm. Math. Phys. 108, 437-467, (1987) 